BRENO BARTH AMARAL DE ANDRADE

\title{
Desempenho visual na correção de miopia com óculos e lentes de contato gelatinosas
}

Tese apresentada ao Departamento de Oftalmologia e Otorrinolaringologia da Faculdade de Medicina da Universidade de São Paulo para obtenção do título de Doutor em Ciências.

Área de concentração: Oftalmologia

Orientador: Prof. Dr. Newton Kara José

Co-orientador: Prof. Dr. Milton Ruiz Alves

São Paulo

2004 
Como é o lugar quando ninguém passa por ele?

Existem as coisas sem ser vistas?

Carlos Drummond de Andrade 
Dedicatória 
Este trabalho é dedicado a todas as pessoas que nos doaram, com carinho e atenção, o estímulo necessário para que o caminho pudesse ser percorrido:

- minha mãe Selma, eterna professora, que nos ensinou os valores morais e éticos que vêm sinalizando o rumo a seguir;

- meu pai Antonio, que nos ensinou que a honestidade é uma virtude incondicional ao homem;

- minha esposa llana que, durante os nossos quinze anos de união, nunca deixou de regar os nossos ideais;

- minha filha Bruna que, com sua presença, vem renovando a esperança no futuro por vir. 
Agradecimentos 
Ao Professor Dr. Newton Kara José, exemplo de liderança, que nos abriu as portas da Universidade de São Paulo permitindo o nosso acesso à carreira universitária.

Ao Professor Dr. Milton Ruiz Alves, exemplo de dedicação ao trabalho, responsável por parte de minha formação científica e pelo constante estímulo e apoio neste nosso estudo.

Ao Professor Dr. Carlos Alberto Rodrigues Alves, por seu incentivo à pesquisa científica e dedicação ao curso de pós-graduação.

Ao Professor Dr. Walton Nosé e Dra. Regina Nosé, que nos receberam como parte da família e cujo ensinamentos e valores estarão sempre em nossa memória.

Ao Professor Eduardo Henrique Silveira de Araújo que, com esmero, analisou os dados estatísticos deste trabalho.

Ao Dr. Marcos Alonso Garcia, por sua inestimável amizade.

Ao Dr. Gustavo Victor de Paula Baptista, companheiro e amigo prestativo.

À Dra. Eleonore Jean Norris, Dra. Andréia Urbano e Dra. Márcia Bentes Sá, pelo carinho e atenção.

À Sra. Maria José Poinho, pelo auxílio nas adaptações das lentes de contato.

À Srta Regina Ferreira de Almeida, por sua amizade e dedicação ao curso de pós-graduação.

Aos amigos da Eye Clinic Day Hospital e do Hospital das Clínicas da Faculdade de Medicina da Universidade de São Paulo, pelo apoio em todas as fases deste estudo.

À Dra. Ilana Barth e ao Dr. Cláudio Luz, amigos e companheiros em todos os momentos. 
À Diana Barth, pela alegria e energia de viver.

Ao llton Miranda e à Telma Miranda, pelo exemplo de união.

Aos pacientes e a todos aqueles que me apoiaram em todas as fases de minha vida. 
Sumário 
Lista de figuras

página

Lista de abreviaturas e símbolos ................................................. xiv

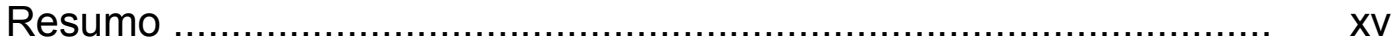

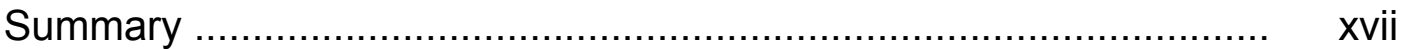

1. INTRODUÇÃO ......................................................................... 1

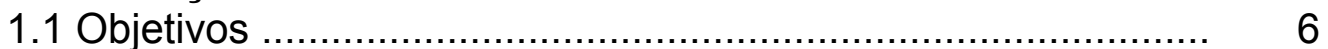

2. REVISÃO DA LITERATURA ..................................................... 7

$2.1 \mathrm{O}$ olho como aparelho dióptrico ....................................... 8

2.1.1 Alterações sofridas pela luz ao atravessar os meios oculares (reflexão, absorção e refração) ...................... 8

2.1.2 Construção da imagem pelo olho ............................... 11

2.2 Lentes oftálmicas (óculos) ............................................... 13

2.2.1 Aberrações ópticas com lentes oftálmicas ................... 14

2.3 Lentes de contato ............................................................ 17

2.3.1 Aberrações ópticas com lentes de contato .................. 17

2.4 Medidas da função visual ...................................................... 19

2.4.1 Acuidade Visual ................................................. 19

2.4.2 Testes de resolução visual .................................... 20

2.4.3 Sensibilidade ao Contraste ....................................... 20

2.4.4 Sistemas de análise de frentes de onda ...................... 21

2.4.4.1 Tipos de sistema de análise de frentes de onda 23

3 CASUÍSTICA E MÉTODOS .......................................................... 27

3.1 Ética e informação aos pacientes ..................................... 28

3.2 Tipo de estudo .............................................................. 29

3.3 Objeto do estudo .......................................................... 29

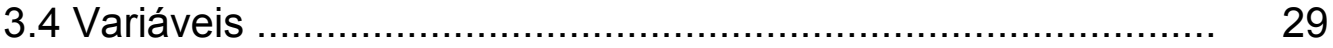

3.4.1 Variáveis dependentes ............................................ 29

3.4.2 Variáveis independentes ........................................ 30

3.4.3 Variáveis com função descritiva ............................. 30

3.5 População e amostra ........................................................... 30

3.5.1 Critérios de inclusão e exclusão .................................. 31

3.6 Materiais .................................................................... 32

3.7 Procedimentos e técnicas .............................................. 32

3.7.1 Técnica de adaptação das lentes de contato
gelatinosas ...................................................... 33

3.7.2 Medida de Acuidade Visual de Alto Contraste ............. 34

3.7.3 Medida de Sensibilidade ao Contraste ........................ 34

3.7.4 Sobre-refração e medida das Aberrações de alta ordem ............................................................. 35

3.8 Instrumentos .............................................................. 36

3.9 Coleta de dados ....................................................... 36

3.10 Processamento dos dados ............................................. 37

3.11 Procedimento para análise dos dados ............................. 37

3.12 Análise estatística ................................................... 37 
3.12.1 Análise descritiva .......................................... 37

3.12.2 Análise comparativa ...................................... 38

4 RESULTADOS ...................................................................... 40

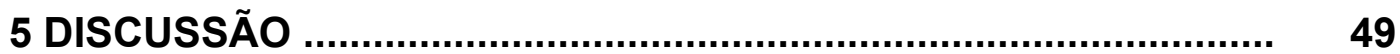

6 CONCLUSÕES ......................................................................... 60

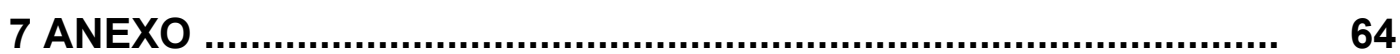

8 REFERÊNCIAS ....................................................................... 66

9 BIBLIOGRAFIA CONSULTADA ................................................. 75 
Listas 


\section{LISTA DE FIGURAS}

Figura 1. Representação gráfica dos valores de sensibilidade ao contraste em unidades logarítmicas nas freqüências 3 , 6,12 e 18 ciclos por grau com os óculos e com as lentes de contato gelatinosas (Acuvue $\circledR$ 2, Biomedics $\circledR$ 55 e Focus ${ }^{\circledR}$ 1-2 week). Eye Clinic Day Hospital, São Paulo - 2003

Figura 2. Representação gráfica dos valores de RMS em micrômetros da aberração de terceira ordem sem e com as lentes de contato gelatinosas (Acuvue ${ }^{\circledR}$ 2, Biomedics ${ }^{\circledR} 55$ e Focus ${ }^{\circledR}$ 1-2 week). Eye Clinic Day Hospital, São Paulo - 2003

Figura 3. Representação gráfica dos valores de RMS em micrômetros da aberração trifoil sem e com as lentes de contato gelatinosas (Acuvue ${ }^{\circledR}$ 2, Biomedics ${ }^{\circledR} 55$ e Focus ${ }^{\circledR}$ 1-2 week). Eye Clinic Day Hospital, São Paulo $-2003$ 


\section{LISTA DE QUADROS E TABELAS}

Quadro 1 Aberrações ópticas produzidas durante o uso de lentes oftálmicas (óculos) e lentes de contato .......................... 19

Quadro 2 Sistemas de Análise de Frente de Onda ..................... 23

Quadro 3 Parâmetros das LCG Acuvue ${ }^{\circledR} 2$, Biomedics ${ }^{\circledR} 55 \mathrm{E}$ Focus $₫ 1$ 1-2 week ................................................. 32

Tabela 1 Dados demográficos e aspectos clínicos dos sujeitos do estudo. Eye Clinic Day Hospital, São Paulo - 2003.

Tabela 2 Resultados da sobre-refração em dioptrias pelo Zywave com as lentes de contato gelatinosas (Acuvue $₫$ 2, Biomedics $₫ 55$ e Focus $₫ 1-2$ week). Eye Clinic Day Hospital, São Paulo - 2003

Tabela 3 Sensibilidade ao contraste, em unidades logarítmicas, e respectivas freqüências espaciais em ciclos por grau, com os óculos e com as lentes de contato gelatinosas (Acuvue $₫$ 2, Biomedics ${ }^{\circledR} 55$ e Focus ${ }^{\circledR} 1-2$ week). Eye Clinic Day Hospital, São Paulo - 2003

Tabela 4 Aberrações de alta ordem representadas pelos polinômios de Zernike em RMS em micrômetros sem e com as lentes de contato gelatinosas (Acuvue ${ }^{\circledR}$ 2, Biomedics ${ }^{\circledR} 55$ e Focus $\AA$ 1-2 week). Eye Clinic Day Hospital, São Paulo - 2003

Tabela 5 Aberrações de alta ordem em RMS em micrômetros, sem e com as lentes de contato gelatinosas (Acuvue ${ }^{\circledR}$ 2, Biomedics ${ }^{\circledR} 55$ e Focus ${ }^{\circledR}$ 1-2 week). Eye Clinic Day Hospital, São Paulo - 2003

Tabela 6 Níveis de aberrações ópticas em RMS em micrômetros, sem e com as lentes de contato gelatinosas (Acuvue $₫ 2$ 2, Biomedics $₫ 55$ e Focus $₫ 1$ 1-2 week). Eye Clinic Day Hospital, São Paulo - 2003 


\section{LISTA DE ABREVIATURAS E SÍMBOLOS}

\begin{tabular}{|c|c|}
\hline$\%$ & porcentagem, por cento \\
\hline$\mu$ (letra grega mi) & micra, plural de micron $\left(10^{-6} \mathrm{~m}\right)$ \\
\hline$\mu \mathrm{m}$ & micrômetro \\
\hline AV & acuidade visual \\
\hline Avcc & acuidade visual com correção \\
\hline cc & com correção \\
\hline Cpg & ciclos por grau \\
\hline csv & curva de sensibilidade ao contraste \\
\hline D & dioptria \\
\hline Dk & $\begin{array}{l}\text { Coeficiente de permeabilidade ao oxigênio do } \\
\text { material da lente de contato }\left(\mathrm{cm}^{2} / \mathrm{s}\right)\left(\mathrm{O}_{2} / \mathrm{ml} \times \mathrm{mmHg}\right)\end{array}$ \\
\hline ed. & editor(es) \\
\hline et al. & e outros autores \\
\hline $\mathrm{F}$ & de Snedcor (Prova de Fisher) \\
\hline FE & freqüência espacial \\
\hline Fig. ou fig. & Figura \\
\hline $\ln$ & $\mathrm{em}$ \\
\hline LASEK & keratomileusis epthelium situ assisted laser \\
\hline Laser & light amplification by stimulated emission \\
\hline LASIK & keratomileusis in situ assisted laser \\
\hline LC & Lente(s) de Contato \\
\hline LCG & Lente(s) de Contato Gelatinosa(s) \\
\hline $\log$ & logaritmo, base 10 \\
\hline $\mathrm{mm}$ & milímetro \\
\hline p. & página \\
\hline p-valor & probabilidade de erro \\
\hline RMS & Root Mean Square, micrômetro \\
\hline Tab. & Tabela \\
\hline
\end{tabular}


Resumo 
Andrade BBA. Desempenho visual na correção de miopia com óculos e lentes de contato gelatinosas [tese]. São Paulo: Faculdade de Medicina, Universidade de São Paulo; 2004. 75p.

Objetivos: Avaliar o desempenho visual, por meio da AV logMAR de alto contraste, sensibilidade ao contraste e análise de frentes de onda de indivíduos portadores de miopia com ou sem astigmatismo, corrigidos com óculos e com três diferentes LCG [Acuvue ${ }^{\circledR}$ (Vistacon J\&J Vision Care Inc., EUA), Biomedics $₫ 55$ (Ocular Science, EUA) e Focus ${ }^{\circledR}$ 1-2 week (Ciba Vision Corporation, EUA)]. Métodos: Realizou-se um estudo clínico e prospectivo com intervenção em amostra constituída por quarenta indivíduos portadores de miopia entre $-1,00 \mathrm{D}$ e $-4,50 \mathrm{D}$, sem ou com astigmatismo até $-0,75 \mathrm{D}$, com AV corrigida com óculos e com as LCG igual ou superior a 20/20. Cada paciente teve um olho escolhido por sorteio para a avaliação do desempenho visual. Os sujeitos foram, inicialmente, submetidos aos seguintes exames: anamnese, exame ocular externo, refratometria dinâmica subjetiva, medida da AVcc, fundoscopia direta, biomicroscopia do segmento anterior e avaliação da relação lente de contato-córnea com as três LCG, quando tiveram prescrição de óculos. Posteriormente, retornaram para a adaptação das LCG, quando foram submetidos à medida de AV logMAR de alto contraste (com óculos e com as LCG), avaliação de sensibilidade ao contraste (com os óculos e com as LCG), sobre-refração objetiva e análise de frentes de onda com o aberrômetro Zywave (Bausch \& Lomb, EUA)(sem e com as LCG). Para a medida da AV logMAR de alto contraste, utilizou-se a tabela de AV do EDTRS (Early Treatment Diabetic Retinopathy). O teste de sensibilidade ao contraste foi realizado com o aparelho CSV-1000 (VectorVision, Ohio, EUA). A sobre-refração com as LCG e as medidas das aberrações de alta ordem foram realizadas com o aberrômetro Zywave. Resultados: A sobre-refração objetiva realizada pelo aberrômetro Zywave detectou diferença significativa entre as LCG Acuvue ${ }^{\circledR} 2$ e Biomedics $® 55$, em relação aos componentes refrativos esfera e equivalente esférico, que se mostraram hipercorrigidos em relação às LCG Focus ${ }^{\circledR}$ 1-2 week. A AV LogMAR e sensibilidade ao contraste não detectaram diferenças no desempenho visual com os óculos e com as três LCG. A análise de frentes de onda detectou diferença significativa na aberração de terceira ordem sem e com as LCG, com melhor desempenho visual com as LCG Acuvue ${ }^{\circledR} 2$ e Biomedics ${ }^{\circledR} 55$. Em relação ao coeficiente Z330, não houve diferença significativa no desempenho visual entre as três LCG, o qual foi superior ao observado sem LC. Em relação ao coeficiente 
Z331, não houve diferença significativa no desempenho visual entre as LCG Acuvue ${ }^{\circledR} 2$ e Biomedics $\circledR 55$, que foi superior ao sem LC e com a LCG Focus $₫$ 1-2 week. Em relação ao polinômio Z440, não houve diferença significativa no desempenho visual entre as três LCG, que foi superior ao sem LC. Em relação ao polinômio Z511, não houve diferença significativa no desempenho visual entre as LCG Biomedics $® 55$ e sem LC, que foi superior ao observado com as LCG Focus $®$ 1-2 week e Acuvue® 2. Em relação ao polinômio Z551, não houve diferença significativa no desempenho visual entre as LCG Acuvue® 2 e sem LC, que foi superior ao observado com as LCG Biomedics ${ }^{\circledR} 55$ e Focus ${ }^{\circledR} 1-2$ week. Em relação ao nível trifoil de aberração de terceira ordem houve diferença significativa entre com e sem LCG, sendo superior o desempenho visual com LCG Acuvue® 2, seguido por Biomedics ${ }^{\circledR} 55$, Focus $®$ 1-2 week e sem LCG. Conclusões: A análise de frentes de onda detectou diferenças no desempenho visual com óculos e com as LCG, sendo, portanto, uma medida mais sensível da função visual que a AV LogMAR de alto contraste e a sensibilidade ao contraste. O modelo de avaliação de desempenho visual com análise de frentes de onda utilizado nesta investigação serve de modelo para pesquisas semelhantes.

Descritores: 1. MIOPIA/terapia 2. ÓCULOS/utilização 3. LENTES DE CONTATO/ estatística e dados numéricos 4. ACUIDADE VISUAL 5. SENSIBILIDADES DE CONTRASTE 6. ESTUDOS PROSPECTIVOS 
Summary 
Andrade BBA. Visual performance in myopic correction with spectacles and soft contact lenses [thesis]. São Paulo: "Faculdade de Medicina, Universidade de São Paulo"; 2004. 75p.

Objective: To evaluate the visual performance (VP) by high contrast visual acuity (VA), contrast sensitivity (CS) and wavefront in myopic patients with or without astigmatism corrected with spectacles and with three different soft contact lenses (SCL) [Acuvue® 2 (Vistacon J\&J Vision Care Inc., USA), Biomedics® 55 (Ocular Science, USA) e Focus $\AA^{1-2}$ week (Ciba Vision Corporation, USA)]. Methods: An interventional prospective clinical trial studied a sample of 40 myopic patients $(-1.00$ to $-4.50 \mathrm{sph}$, with or without astigmatism up to $-0.75 \mathrm{cyl}$ ) with VA 20/20 or better corrected by spectacles or SCL. Each patient had one eye randomized to VP evaluation. The following were performed initially: general history, external ocular exam, subjective dynamic refractometry, VA measurement, direct ophthalmoscopy, anterior segment slit-lamp biomicroscopy and $\mathrm{SCL}$ adaptation with three different SCL. Patients then returned for SCL adaptation and high contrast VA measurement (with spectacles and $\mathrm{SCL}$ ), objective over refraction, $\mathrm{CS}$ test and wavefront analysis (with and without SCL). For high contrast VA, LogMAR ETDRS VA table was used. The CS test was done with CSV 1000 (Vector Vision, Ohio, USA) instrument. The over refraction with $\mathrm{SCL}$ and high order aberration measurement was performed with Zywave ${ }^{\circledR}$ aberrometer (Bausch \& Lomb, USA). Results: The Zywave aberrometer objective over refraction detected a significant difference between Acuvue $\circledast 2$ and Biomedics $\circledast 55$ regarding spheric refractive components and spheric equivalent. Both SCL showed hypercorrection comparing to Focus 1-2 week. Visual performance was not significantly different with spectacles and the three SCL in VA and CS measurements. The wavefront analysis detected a significant difference in a third order aberration with and without SCL, with better VP in Acuvue $₫ 2$ and Biomedics $® 55$. Visual Performance was not significantly different in Z330 and Z440 coefficients among the three SCL. They were superior when compared to without SCL. Visual Performance was not significantly different in Z331 coefficient between Acuvue $\AA 2$ and Biomedics $\AA$ 55. It was superior when compared to no SCL and with Focus 1-2 week. Visual Performance was not significantly different in Z511 coefficient between Biomedics $₫ 55$ and no SCL. It was superior when compared to Focus $\AA$ 1-2 week and Acuvue $₫$ 3. Visual Performance was not significantly different in Z551 coefficient between Acuvue ${ }^{\circledR} 2$ and no SCL. It 
was superior when compared to Biomedics ${ }^{\circledR} 55$ and Focus ${ }^{\circledR} 1-2$ week. Visual Performance was significantly different in trefoil coefficient between with and without SCL. Visual Performance was superior with Acuvue ${ }^{\circledR} 2$ when compared to Biomedics ${ }^{\circledR} 55$ and Focus ${ }^{\circledR}$ 1-2 week and without SCL. Conclusion: In VP evaluation with spectacles and SCL the wavefront analysis was a more sensible measurement of visual function when compared to high contrast VA and CS. The evaluation model of VP with wavefront analysis developed in this investigation may be useful for further similar studies.

Keywords: 1. MYOPIA/therapy 2. SPETACLES/utilisation 3. CONTACT LENSES/ statistics and numeric data 4. VISUAL ACUITY 5. SENSIBILITIES OF CONTRAST 6. PROSPECTIVE STUDIES 
1 Introdução 
O sistema óptico do olho humano produz uma variedade de aberrações que resultam em distorção na imagem retínica (Tunnacliffe, 1987).

As aberrações cromáticas têm origem na dispersão da luz que ocorre nos elementos refrativos oculares (Collins et al., 1995). As aberrações monocromáticas estão relacionadas com um comprimento de onda da luz e decorrem do fato de que lentes reais (lentes dos óculos, LC ou elementos refrativos do bulbo ocular) modificam frentes de ondas planas incidentes, de modo que os raios paracentrais acabam alterados diferentemente dos periféricos, causando a não coincidência com um ponto focal único (Collins et al., 1995; Jankov et al., 2002). As aberrações monocromáticas constituem a mais significativa variável que afeta o poder de resolução do olho sadio (Applegate et al., 2003). O esforço despendido na correção dessas aberrações ópticas visa a melhorar o desempenho do sistema óptico ocular e a formar uma imagem retínica mais nítida. Todo o processamento dessa imagem, na retina e nos outros locais do sistema nervoso central, é responsável por elevar os limites da percepção visual humana (Liang et al., 1997; Williams et al., 2000; Applegate et al., 2003; Chamon et al., 2003). 
A avaliação da qualidade do sistema óptico ocular refere-se, portanto, à avaliação da imagem retínica de um objeto. A acuidade visual (AV) representa a faculdade que possui o olho de distinguir dois ou mais pontos, mais ou menos aproximados e situados no mesmo plano, perpendicular ao eixo visual (Prado, 1983). Usualmente, a visão é mensurada por meio de testes de $\mathrm{AV}$ que determinam o menor detalhe que pode ser medido usando letras ou símbolos de cor preta contra um fundo branco, com quase $100 \%$ de contraste (Bailey, 1998). Entretanto, a maior parte dos objetos, observados em um ambiente, revelam níveis de contraste bastante inferiores, exigindo a percepção de pequenas variações de luminosidade entre regiões que não apresentam bordas nítidas de separação. A capacidade do sistema visual para lidar com essas variações espaciais da percepção caracteriza a sensibilidade ao contraste espacial (Elliott, 1998). Os testes de sensibilidade ao contraste espacial medem a capacidade de detectar diferenças entre áreas de diversas luminâncias, situadas lado a lado no espaço, possibilitando uma avaliação mais precisa da função visual do que os testes de AV com os optotipos (Elliott, 1998). A função de transferência de modulação (do inglês MTF, Modulation Transfer Function) representa qual a perda de contraste de uma imagem ao passar por um sistema óptico. A modulação de um objeto ou imagem é o contraste medido por uma curva senoidal que tem, nas ordenadas, a intensidade de luz; portanto, quanto mais claro (branco), mais alta será a ordenada de um ponto e quanto mais escuro (preto), mais baixa será sua ordenada. A diferença entre o ponto mais alto e o ponto mais baixo da senóide 
representa o contraste do objeto ou da imagem. A modulação (ou contraste) de um objeto será transferida a uma imagem pelo sistema óptico. Quanto melhor for o sistema óptico, mais semelhantes serão os contrastes do objeto e da imagem. Em sistema real, portanto imperfeito, o preto de um objeto não será tão "preto" em uma imagem desse objeto, o mesmo acontecendo com o branco que se tornará acinzentado. Dessa maneira, o contraste da imagem será sempre menor do que a do próprio objeto (Chamon et al., 2003).

Os instrumentos de análise de frentes de onda (ou aberrômetros) são aparelhos que medem a refração ocular e o desvio de um pequeno feixe luminoso ao passar por todo o sistema óptico (Doane et al., 2001). As aberrações de baixa ordem são aquelas passíveis de correção com óculos (miopia, hipermetropia e astigmatismo). As que não são passíveis de correção com lentes esferocilíndricas constituem as aberrações de alta ordem (Chamon et al., 2003). Até o advento da ablação personalizada a laser, o tratamento das aberrações de alta ordem só podia ser feito com lentes de contato (LC) rígidas ou, em alguns casos, por meio de incisões arqueadas. A análise de frentes de onda tornou-se uma ferramenta necessária para a identificação e tratamento das aberrações de alta ordem (Mrochen et al., 2000; Williams et al., 2000; Argento, Cosentino, 2001; Manns et al., 2001; Marcos et al., 2001; Moreno-Barriuso et al., 2001; Rudy et al., 2002; Aizawa et al., 2003).

A correção de erros refrativos com a colocação de lentes oftálmicas (óculos) na frente dos olhos de um indivíduo altera o seu mundo visual. As imagens borradas deverão tornar-se nítidas e poderão parecer estar em 
distâncias distintas daquelas dos objetos originais. As imagens poderão estar aumentadas ou diminuídas, e efeitos prismáticos poderão modificar a posição aparente dos objetos. As aberrações produzidas pelas lentes também poderão alterar a forma e a nitidez das imagens (Stephens, 1998). No momento atual, as LC ainda constituem a melhor forma de correção óptica dos erros refrativos, oferecendo ao indivíduo a melhor qualidade visual e excedendo qualquer outra modalidade corretiva no campo da oftalmologia (Benjamin, 1998).

Estudos baseados em análises de frentes de onda mostraram que as aberrações oculares aumentam significativamente após cirurgia refrativa. O principal motivo de insatisfação após cirurgia refrativa (PRK, LASIK ou LASEK) é a deterioração da AV em condições mesópicas, mostrando que o aumento das aberrações oculares torna-se mais proeminente em baixa luminosidade, quando o diâmetro pupilar aumenta (Chalita et al., 2003). O tratamento personalizado dos erros refrativos com Excimer laser é um novo conceito, em desenvolvimento, de cirurgia refrativa, o qual se baseia na detecção e na posterior correção das aberrações oculares de baixa e alta ordem, identificadas pela análise de frentes de onda. Esse novo procedimento apresenta potencial para a obtenção de acuidade visual superior à alcançada com óculos ou LC (Chalita et al., 2003).

Atualmente, muito esforço e tempo são gastos avaliando-se alterações de $\mathrm{AV}$, sensibilidade ao contraste e aberrações de alta ordem em indivíduos submetidos a tratamentos fotoablativos convencionais e personalizados. Na literatura pesquisada, não se encontraram estudos 
comparando o desempenho visual da correção de erros refrativos com óculos e LCG disponíveis comercialmente, considerando-se as aberrações de alta ordem. A criação de um banco de dados a partir de investigações realizadas nessa área permitirá fornecer subsídios que poderão contribuir para melhorar a qualidade óptica e o desempenho visual da correção do erro refrativo com lentes oftálmicas e com LCG.

\subsection{Objetivos}

Avaliar o desempenho visual, por meio da AV logMAR de alto contraste, sensibilidade ao contaste e análise de frentes de onda de indivíduos portadores de miopia com ou sem astigmatismo, corrigidos com óculos e com três diferentes LCG [Acuvue® 2 (Vistacon J\&J Vision Care Inc., EUA), Biomedics $₫ 55$ (Ocular Science, EUA) e Focus $₫$ 1-2 week (Ciba Vision Corporation, EUA)]. 
2 Revisão da literatura 


\subsection{O olho como aparelho dióptrico}

Estudar o olho como aparelho dióptrico é compará-lo a um sistema dióptrico centrado, dentro das condições de aproximação de Gauss, a fim de se conhecerem as diversas características das imagens formadas pelo mesmo (Prado, 1983).

2.1.1 Alterações sofridas pela luz ao atravessar os meios oculares (reflexão, absorção e refração)

Cerca de $2,5 \%$ da luz que chega ao olho é refletida pela córnea e filme lacrimal, permitindo a visualização das imagens de objetos colocados diante da córnea (Prado, 1983). Pelo estudo do tamanho dessas imagens, calculou-se o raio de curvatura dos diferentes meridianos da face anterior da córnea, meio empregado na determinação do astigmatismo corneano (Horner et al., 1998). As imagens dadas pela superfície anterior da córnea são denominadas imagens de primeira ordem. São imagens especulares, diretas, virtuais e menores do que o objeto porque formadas por espelho convexo (Prado, 1983). 
As demais superfícies refringentes do olho (posterior da córnea, anterior e posterior do cristalino e, em dadas condições, anterior e posterior do núcleo deste) também dão imagens especulares, menos nítidas que as primeiras que são vistas após refração, em sentido oposto ao da luz que as formou. São imagens de segunda ordem, diretas quando formadas por superfícies côncavas (face posterior da lente e do núcleo) (Prado, 1983). Segundo Prado (1983) deve-se a Purkinge e Sanson o estudo dessas imagens que evidenciaram a existência do cristalino e revelaram, pela alteração de tamanho, seus diferentes movimentos durante a acomodação.

Afastando-se o olho das condições de absoluta emetropia, é natural que parte da luz que transita em seu interior não alcance a retina. Decorre esse fato de reflexões elementares determinadas pela própria estrutura da matéria viva (Prado, 1983).

O filme lacrimal e o epitélio corneano - cujos índices de refração são, respectivamente, 1,416 e 1,376 em condições normais - pouco interferem no fenômeno de refração (Katz, 1981).

A curvatura da face anterior da córnea é que tem a maior importância no estudo da refração ocular. Suas primeiras medidas foram obtidas in vivo e mediante processo indireto de determinação do tamanho de imagem, dada pela sua face anterior. A dificuldade inicial do método resultou da impossibilidade de manter fixo o olho vivo; por esse motivo, aplicou-se o princípio do desdobramento da imagem empregado em Astronomia para a determinação do diâmetro aparente dos astros (Prado, 1983). Segundo 
Horner (1998), esse método foi aperfeiçoado por Helmholtz no seu oftalmômetro e, posteriormente, no oftalmômetro de Javal-Schioetz.

A córnea possui duas zonas perfeitamente distintas: uma zona central (zona óptica), medindo entre 4 e $6 \mathrm{~mm}$ de diâmetro e de curvatura sensivelmente esférica, e outra periférica, mais achatada. O comprimento do raio de curvatura da superfície anterior da córnea apresenta um valor entre 7,2 e $8,6 \mathrm{~mm}$. Apresenta-se menor o raio de curvatura da face posterior que mede, aproximadamente, 7,0 mm (Alves, 2003).

O índice de refração do humor aquoso e do humor vítreo é considerado igual a 1,336 (Katz, 1981).

O cristalino é uma lente biconvexa que tem o raio de curvatura da face anterior (em repouso) de cerca de $10 \mathrm{~mm}$ e o da face posterior, de 6 $\mathrm{mm}$. Seu diâmetro mede, aproximadamente, $10 \mathrm{~mm}$ e sua espessura, entre os pólos, 3,6mm. A face anterior da lente, em repouso dista cerca de 3,6 mm da face posterior da córnea e a posterior, 7,2 mm (Prado, 1983). Segundo Prado (1983), Gullstrand encontrou 1,386 para o índice de refração do córtex e 1,406 para o núcleo do cristalino.

Conhecidas de modo aproximado as constantes dos componentes dióptricos do olho, considerou-se o sistema formado por três dioptros centrados, a saber: superfície corneana separando o ar de um meio de índice comum (humor aquoso e humor vítreo), superfície anterior e superfície posterior do cristalino, ambas imersas em meios de índices iguais (Katz, 1981). 
A córnea representa um dioptro esférico valendo cerca de 43 D; o cristalino, aproximadamente $19 \mathrm{D}$ (em repouso); o olho esquemático, um valor de 58 D (Prado, 1983).

Segundo Katz (1981), após a publicação de Listing (1853) apresentando o olho reduzido, apareceu o de Donders (1864), ainda mais simplificado e no qual só existe uma superfície dióptrica entre o meio ocular e o ar. Segundo Prado (1983), Gullstrand realizou cálculos mais precisos para o olho reduzido, considerando-o um dioptro esférico de raio de curvatura igual a 5,73 mm separando dois meios de índices diferentes. Sua superfície ficaria $1,35 \mathrm{~mm}$ atrás da córnea. $\mathrm{O}$ ponto nodal ficaria $7,08 \mathrm{~mm}$ atrás da córnea. A distância focal anterior estaria 15,7 mm diante da córnea e a distância focal posterior, $24,13 \mathrm{~mm}$ atrás da córnea.

\subsubsection{Construção da imagem pelo olho}

Aceita-se como ângulo mínimo de resolução visual o ângulo de um minuto formado no ponto nodal pelos raios extremos que limitam o objeto no espaço. Esse ângulo corresponde, na retina, a um afastamento de 0,004 mm, pouco mais que o diâmetro dos cones, na fóvea (Katz, 1981).

Como aparelho dióptrico, o olho apresenta defeitos que são comuns aos dioptros, em geral. Entre os principais defeitos fisiológicos, destacam-se: a difração da luz, a aberração cromática, a aberração esférica, a 
descentração, a aberração periférica e os círculos de difusão (Katz, 1981; Benjamin, 1998; Stephens, 1998).

Quando uma imagem luminosa atravessa um orifício de pequenas dimensões, o ponto central dessa pequena imagem aparece circundado de círculos alternadamente claros e escuros. A pupila em miose intensa concorre para o seu aparecimento (Katz, 1981).

A luz branca sendo composta de radiações luminosas de diferentes comprimentos de onda, os raios que mais desviam, durante a refração ocular, são os de menor comprimento de onda, o que equivale a dizer, considerando-se que a retina se encontra no foco dos raios de médio comprimento de onda, que o olho deveria ser míope para as radiações azuis e hipermétrope para as vermelhas. É, todavia, a descentração da fóvea que contribui para a diminuição dessa aberração cromática (Prado, 1983).

A aberração de esfericidade é inerente às lentes espessas, como o cristalino, quando a luz as atravessa pelas margens. $O$ diafragma pupilar intercepta os raios periféricos diminuindo seu efeito (Prado, 1983).

O olho apresenta certo grau de descentração, ficando o centro óptico da córnea $1 / 4$ de $\mathrm{mm}$ abaixo dos centros da face anterior e posterior do cristalino. O plano que contém o equador do cristalino não é perpendicular ao eixo do sistema. A fóvea está deslocada cerca de 1,25 mm para o lado temporal da extremidade posterior do eixo ântero-posterior do bulbo ocular. Assim, resulta que o eixo visual faz, com o eixo anatômico, um ângulo com vértice no ponto nodal (Benjamin, 1998). 
A aberração periférica origina-se das imagens formadas na periferia da retina e que apresentam certa inferioridade na nitidez, conhecida como distorção e coma ou astigmatismo radial (Benjamin, 1998).

Quando um determinado objeto é fixado, os outros objetos que se encontram imediatamente antes ou depois dele formam também suas imagens na retina, à custa de círculos de difusão (Benjamin, 1998).

Ao lado dos defeitos fisiológicos acima descritos, o olho apresenta certos defeitos ou erros de refração que, em geral, são decorrentes de alteração no comprimento do eixo ântero-posterior ou de alteração de curvatura da córnea.

\subsection{Lentes oftálmicas (óculos)}

As lentes oftálmicas representam meios transparentes, homogêneos, de índice de refração entre 1,523 e 1,80, limitados por superfícies curvas ou, associadamente, plana e curva. Em cada espécie de lente (esférica ou cilíndrica), reconhecem-se dois tipos diferentes: convergentes e divergentes. As primeiras apresentam as bordas finas e o centro espesso e as segundas, ao contrário, o centro fino e as bordas espessas (Stephens, 1998). 
Uma lente oftálmica corrige um erro refrativo se o seu ponto focal secundário coincide com o ponto remoto do olho, com a acomodação relaxada (Prado, 1983).

\subsubsection{Aberrações ópticas com lentes oftálmicas (óculos)}

Várias são as espécies de aberrações, indicadas abaixo, que ocorrem nas lentes oftálmicas (Prado, 1983).

a) Aberração esférica, que tem como causa a incidência marginal de raios luminosos.

b) Aberração astigmática, que representa o resultado da aberração esférica considerada simultaneamente, em dois planos perpendiculares.

c) Aberração cromática, que representa a dispersão da luz como conseqüência da sua própria composição.

d) Distorção, que é dada pelas lentes ao formarem imagens de objetos cujos pontos distam diferentemente do plano da lente.

e) Curvatura de imagem (ou campo), que ocorre devido ao inevitável astigmatismo de incidência oblíqua, onde a imagem de um objeto plano apresenta sempre uma curvatura.

Existem cinco aberrações monocromáticas quando a luz monocromática está presente (Stephens, 1998). Delas, as menos importantes para as lentes oftálmicas são a aberração esférica e coma, que 
têm efeitos desprezíveis na qualidade óptica da imagem (Stephens, Davis, 1993). De maior importância para as lentes oftálmicas, são a aberração astigmática e a de curvatura de imagem (Stephens, 1998). A aberração distorção resulta de alteração na magnificação da imagem do centro para a periferia da lente (Morgan, 1978; Katz, 1981).

Existem duas aberrações cromáticas (aberração cromática longitudinal e transversa ou lateral) que são o resultado de dispersão, conforme a variação do índice de refração com o comprimento de onda que ocorre com todos os materiais (Stephens, 1998). A medida mais comum de dispersão de lentes oftálmicas é o número de Abbe, também conhecido como valor óptico de constrigência do material (Stephens, 1998).

Dado que um determinado desenho da lente pode corrigir astigmatismo radial ou curvatura de imagem, mas não ambas ao mesmo tempo, que aberração deveria então ser corrigida? Trata-se de uma questão que vem sendo debatida há muito tempo, e ainda existe pouca evidência de que a correção de uma aberração resulta em lente com qualidade óptica superior. A maioria dos desenhos de lentes atuais promove correções parciais de todas as aberrações (Bennett 1965-a; Bennett 1965-b; Davis, 1973; Atchinson, 1984; Atchinson, 1985). As lentes disponíveis comercialmente têm seus desenhos com ênfase na correção do astigmatismo radial (Stephens, 1998). Em 1926, a American Optical patenteou um desenho em que o astigmatismo radial e a curvatura de imagem encontravam-se dentro de níveis específicos de tolerância, usualmente menos do que $\pm 1 / 8 \mathrm{D}$, para a maioria dos poderes de lentes 
usados (Stephens,1998). A Bausch \& Lomb, em 1929, desenvolveu as lentes Orthogon, que enfatizavam a correção do astigmatismo radial mas permitiam algum erro para que um sistema de curva base mais curvo pudesse ser empregado (Stephens, 1998). Novos desenhos de lentes foram progressivamente introduzidos, como o American Optical Tillyer Masterpiece lens (Stephens, Davis, 1993). As lentes dessa série foram redesenhadas em 1971 (Masterpiece II) (Stephens, Davis, 1993). Novas modificações ocorreram nas lentes oftálmicas com o emprego de novos materiais e desenhos, criando-se as lentes de desenho asférico. As principais vantagens dessas lentes em relação àquelas de desenho não asférico são, para as lentes positivas, a diminuição da espessura central, do peso e do volume da lente, assim como a diminuição da magnificação dos olhos do usuário e a diminuição da magnificação do campo visual do sujeito. Em relação às lentes negativas, destaca-se a diminuição da espessura das bordas e do peso da lente (Stephens, 1998).

As lentes oftálmicas são produzidas tanto de vidro como de plástico. A partir de 1960, preponderaram as lentes fabricadas com material plástico. As vantagens do plástico sobre o vidro incluem diminuição do peso, maior facilidade de tintura, resistência ao impacto (sem necessidade de tratamentos especiais) (Stephens, 1998). Os índices de refração do material vidro variam de 1,523 a 1,80 (vidros de alto índice). Entre eles, o vidro Crown $(n=1,523)$ apresenta o número de Abbe de 59 e, se não fosse por seu peso e pela questão de segurança, permaneceria sendo o padrão-ouro (Stein et al., 1999). Os plásticos mais utilizados na confecção das lentes são 
o CR-39 e o policarbonato. O CR-39 tem metade do peso do vidro Crown, número Abbe 58, índice de refração de 1,49 e é mais resistente ao impacto que as lentes de vidro. As lentes de policarbonato têm índice de refração de 1,59 e apresentam um número de Abbe de 32 (baixo), sendo importante a dispersão nas lentes cujo poder excede a $\pm 5,00 D$ (Stephens, 1998; Stein et al., 1999).

\subsection{Lentes de contato}

A LC é tratada, em termos de óptica geométrica, como uma lente espessa, a despeito do fato de apresentar uma espessura fina (Benjamin, 1998).

\subsubsection{Aberrações ópticas com lentes de contato}

A visão, no uso de qualquer forma de correção óptica, inclusive de LC, é influenciada por todas as aberrações ópticas (Benjamin, 1998). Durante o uso de LC, os efeitos de aberrações inerentes sobre os objetos fora do eixo óptico da LC são mínimos pelo fato da LC acompanhar a linha visual durante os movimentos oculares. Na correção com óculos, essas aberrações "fora do eixo" podem influenciar a visão central quando os olhos 
estão rodados para fora dos centros ópticos das lentes oftálmicas (Benjamin, 1991). No quadro 1, encontram-se as aberrações cromáticas e monocromáticas produzidas durante o uso de lentes oftálmicas (óculos) e LC.

A imagem da retina do olho corrigido com LC, similar ao que ocorre com o olho corrigido com óculos, sofre aberrações "fora do eixo" que afetam a visão periférica (Benjamin, 1991; Benjamin 1998). O uso de óculos e LC permite que aberrações fora do eixo afetem a visão central (Benjamin, 1991; Benjamin, 1998). A córnea, achatada na periferia, tende a reduzir a aberração esférica, mas, quando cobre a periferia da córnea, a LC incrementa a aberração esférica (os raios de luz periféricos encontram poder refrativo maior do que os raios paraxiais), (Benjamin, 1998). As LC de superfície anterior asférica podem oferecer melhor qualidade óptica pois suas periferias podem ser mais achatadas e induzir menor aberração esférica. Isso possibilitará, no futuro, o desenho de LC em que as suas superfícies tanto corrigirão aberrações ópticas como aberrações presentes nos elementos refrativos do olho. As LC com superfície anterior elíptica, por exemplo, reduziriam ou eliminariam a aberração esférica (Benjamin, 1998). 
Quadro 1. Aberrações ópticas produzidas durante o uso de lentes oftálmicas (óculos) e lentes de contato*

\begin{tabular}{|c|c|c|c|}
\hline \multirow{2}{*}{ Aberração } & \multirow{2}{*}{$\begin{array}{l}\text { Posição do } \\
\text { objeto }\end{array}$} & \multicolumn{2}{|c|}{ Déficit visual } \\
\hline & & Óculos & Lentes de Contato \\
\hline Aberração esférica & No eixo & Central & Central \\
\hline Coma & Fora do eixo & Central e periférica & Periférica \\
\hline Astigmatismo radial & Fora do eixo & Central e periférica & Periférica \\
\hline $\begin{array}{l}\text { Curvatura de } \\
\text { imagem }\end{array}$ & Fora do eixo & Central e periférica & Periférica \\
\hline Distorção & Fora do eixo & Central e periférica & Periférica \\
\hline $\begin{array}{l}\text { Aberração } \\
\text { cromática }\end{array}$ & $\begin{array}{l}\text { No e fora do } \\
\text { eixo }\end{array}$ & Central e periférica & Central e periférica \\
\hline $\begin{array}{l}\text { Dispersão } \\
\text { prismática }\end{array}$ & Fora do eixo & Central e periférica & Periférica \\
\hline
\end{tabular}

*Benjamin WJ. Visual optics of contact lens wear. In: Bennets ES, Eissman BA, ed., Clinical Contact Lens Practice, Philadelphia, JB Lippincott, 1991, p. 1-42.

\subsection{Medidas da função visual}

\subsubsection{Acuidade Visual}

A AV representa a capacidade de resolução espacial do sistema visual (Bailey, 1998). Tal capacidade é expressa em tamanho angular de resolução do detalhe que pode ser visualizado pelo observador. Os limites da AV são impostos por fatores ópticos e neurais ou pela combinação de ambos (Bennet, Rabbetts, 1989). 


\subsubsection{Testes de resolução visual}

Uma variedade de diferentes testes de medida de desempenho visual mensura algum aspecto dos limites da capacidade que o sistema visual tem de discernir detalhes ou de reconhecer alvos específicos. Incluem-se os testes de resolução do mínimo detectável, do mínimo separável e de reconhecimento (Bailey, 1998). A maioria dos testes de AV é de reconhecimento e determina quais os menores símbolos, letras ou palavras que podem ser identificados corretamente. O teste de Snellen usa letras como optotipos. A anotação da AV pode ser expressa pela fração de Snellen, notação decimal, menor ângulo de resolução (MAR), logaritmo do menor ângulo de resolução (logMAR), entre outras formas (Bailey, 1998).

\subsubsection{Sensibilidade ao contraste}

A sensibilidade ao contraste é uma medida mais sensível da função visual que a AV de alto contraste (Wolkstein et al., 1980, Sekuler et al., 1986; Wachler et al., 1999). Os testes de sensibilidade ao contraste espacial medem a capacidade de detectar diferenças entre áreas de diferentes luminâncias, situadas lado a lado no espaço, possibilitando uma avaliação mais precisa da AV espacial do indivíduo do que os testes de mensuração de AV com os optotipos (Bailey, 1998). 
Para a aplicação do teste de sensibilidade ao contraste, utiliza-se tabela com um padrão de alternância de barras claras e escuras, chamadas ciclos, que se fundem gradualmente. O número de ciclos por grau de ângulo visual na retina do observador é denominado freqüência espacial (FE). Cada FE é testada, apresentando-se o mesmo padrão em níveis de contraste decrescentes, ou "sombras da cor cinza". Para todas as FE testadas, um limiar de contraste é estabelecido no ponto onde o padrão é levemente perceptível, o qual é anotado e comparado aos valores normativos. Uma curva de sensibilidade ao contraste (CSV) é, então traçada (Bailey, 1998).

\subsubsection{Sistemas de análise de frentes de onda}

A tecnologia de análise de frentes de onda foi originalmente desenvolvida com o objetivo de ser aplicada na Astronomia (Howland, 2000; Doane et al, 2001). Posteriormente, os programas de "Guerra nas Estrelas" e de defesa aeroespacial desenvolveram diversos estudos nessa área. Tal tecnologia foi empregada com o objetivo de analisar as distorções ópticas decorrentes da passagem da luz na atmosfera, ou por outro sistema óptico complexo. Com o conhecimento das aberrações ópticas, foi possível desenvolver sistemas de compensação ou correção que foram acoplados aos telescópios científicos de modo a melhorar os padrões das informações obtidas de estruturas existentes no espaço. A óptica adaptável constitui um sistema óptico fechado, composto de microlupas ou espelhos deformáveis, o qual pode, por exemplo, identificar a imagem de duas estrelas que, através 
de telescópios tradicionais, seriam observadas como apenas uma imagem borrada (Howland, 2000; Doane et al, 2001).

Segundo Howland (2000), esses mesmos métodos de análise de frentes de onda foram desenvolvidos e adaptados para a compensação das aberrações ópticas do olho humano. O primeiro método foi introduzido por Hartmann em 1920. Segundo Doane et al. (2001), Shack, em 1971, criou uma nova versão do sensor Hartmann, a que chamou sistema ShackHartmann. Esse novo sistema tem a capacidade de melhorar a resolução espacial dos objetos, identificando as aberrações ópticas que limitam o desempenho visual do olho humano (Thibos, Hong, 1999; Thibos, 2000).

Para a representação de todas as aberrações detectadas pela análise de frentes de onda, Zernike (1888-1966) descreveu fórmulas matemáticas que permitem a caracterização das aberrações ópticas por meio de polinômios, conhecidos como polinômios de Zernike (Doane et al., 2001).

Os estudos iniciais de Hartmann foram aperfeiçoados por Shack que desenvolveu os primeiros modelos capazes de analisar as aberrações ópticas. Com isso, o sistema passou a ser chamado Hartmann-Shack (Doane et al., 2001). Em 1994, esse mesmo sistema de análise de frentes de onda foi clinicamente adaptado para a oftalmologia (Liang et al., 1994). Liang et al. (1994) demonstraram que a utilização de sistemas de óptica adaptável poderia melhorar a sensibilidade ao contraste e possibilitar melhor qualidade visual, a partir dos conhecimentos da análise de frentes de onda e de sua correção (Liang et al., 1997). 


\subsubsection{Tipos de sistema de análise de frentes de onda}

No momento atual, há diversos sistemas disponíveis para a análise de frentes de onda. Basicamente, são divididos em sistemas de óptica de saída e de entrada. Os sistemas de óptica de entrada estudam as aberrações ópticas do feixe de luz projetado na retina. Os sistemas de óptica de saída avaliam a frente de onda que sai do olho a partir de um feixe de luz coerente, que fora projetado na retina e refletido (Jankov et al., 2003). Com isso, classificam-se os aparelhos de acordo com o seu padrão de funcionamento (Quadro 2).

\section{Quadro 2 - Sistemas de análise da frente de onda}

1. Sistemas de Óptica de Saída

- Sensor Hartmann-Shack

2. Sistemas de Óptica de Entrada

2.a. Sistemas de Imagem Retínica

- Aberrômetro de Tscherning

- Ray Tracing

2.b. Sistemas de Passagem Dupla

- Esquiascopia em Fenda

2.c. Sistemas Subjetivos Ajustáveis

- Refratometria Espacial

O método de análise óptica de saída por meio do sistema HartmannShack é, atualmente, o mais utilizado. Como exemplos, podem ser citados o Alcon (Fort Worth, TX), Bausch \& Lomb (Rochester, NY), VISX (Santa Clara, CA) e Zeiss-Meditec (Dublin, CA). Esse sistema utiliza um feixe de laser 
(diodo) que é direcionado para a retina (mácula), sendo, a seguir, refletido de volta para a pupila, passando pelo vítreo, cristalino, pupila, câmara anterior e córnea. Qualquer aberração óptica criada por essas estruturas irá determinar uma modificação específica no feixe de luz. Após a saída do olho, esse raio de luz atravessa um sistema de lentículas que concentram a frente de onda que será representada por uma grade de pontos. Essas informações são capturadas por um sistema de vídeo de modo que as imagens serão analisadas por comparação com uma frente de onda plana, livre de aberrações. As diferenças entre o feixe capturado e a frente de onda plana representarão as aberrações do olho. A imagem de comparação será codificada e decomposta por meio de análises matemáticas, baseadas nos polinômios de Zernike e traduzidas em mapas de cores (Doane et al., 2001).

Os sistemas ópticos de entrada são caracterizados pela formação da imagem na retina. Trata-se de um sistema desenvolvido, inicialmente, por Tscherning (1894). Howland e Howland, em 1970, descreveram uma utilização prática para o aberrômetro de Tscherning, acoplando lentes de cilindro cruzado para a formação da imagem em nível retínico. Com isso, também foi possível monitorar, subjetivamente, as aberrações monocromáticas do olho humano. Mais tarde o sistema foi aperfeiçoado e essa tecnologia vem sendo empregada por Wavelight (Erlangen, Alemanha) e Schwind (Kleinostheim, Alemanha). Nessa tecnologia, uma grade de pontos é projetada na retina e nela estão representadas todas as distorções induzidas nos feixes de luz que passaram, respectivamente, pela córnea, cristalino e vítreo. A imagem formada na retina é registrada por meio de uma 
câmera CCD de alta sensibilidade, seguindo o mesmo princípio da oftalmoscopia indireta. A partir dessa imagem capturada, sistemas de interpretação utilizam imagens padrão (sem aberrações) para determinar as aberrações existentes. Portanto, a frente de onda analisada é obtida por meio de feixes de luz que entram no olho e se projetam na retina, caracterizando o sistema óptico de entrada (Howland, Howland, 1977).

Além desse sistema de Tscherning, há um outro, semelhante, que utiliza o princípio de ray-tracing que também representa um sistema óptico de entrada. Essa tecnologia vem sendo empregada pela Tracey Technologies (Bellaire, TX). Nesse caso, um conjunto de feixes de laser é disparado em direção à retina de forma paralela. Cada feixe independente passa pela córnea e demais estruturas intra-oculares de modo a chegar à retina. Essa localização é determinada por meio de fotodetectores, permitindo uma análise refrativa de cada ponto isoladamente. Cada feixe é projetado de forma isolada e sua imagem é captada individualmente. Hoje, esse sistema vem sendo utilizado apenas com fins diagnósticos, havendo um acordo com a VISX de exclusividade na criação de uma plataforma para fotoablação corneana personalizada (Molebny et al., 2000).

Outro sistema óptico de entrada, utilizado pela empresa Nidek (Gamagori, Japão), baseia-se na retinoscopia de Coopland. Há, basicamente, um sistema para projeção e outro para recepção. São gerados feixes de luz em fenda, os quais passam por um sistema de lentes e espelhos e atingem a retina. A seguir, esses feixes são refletidos e captados no nível da córnea. A diferença do tempo de chegada e o sentido do feixe 
serão proporcionalmente convertidos em poder refrativo. Ambos os sistemas de projeção e recepção giram sincronicamente em torno do eixo, de modo a medir a refração em cada meridiano isoladamente. Com isso, as informações são obtidas por escaneamento de uma fenda, caracterizando uma dupla passagem dos feixes de luz. A Nidek (OPD Scan) conta com um sistema que processa, de forma conjunta, as informações obtidas com a análise de frentes de onda e com a videoceratoscopia computadorizada, obtida por meio dos discos de Plácido. O aberrômetro pode ser acoplado ao laser Nidek EC-5000 para ablação personalizada (MacRae e Fujieda, 2000).

Smirnov (1961) descreveu outro sistema de análise de frente de onda, baseando-se na refratometria espacial, que inclui um ajuste subjetivo das aberrações oculares. Tal método também representa um sistema óptico de entrada. Essa tecnologia envolve uma abordagem subjetiva fornecida pelo paciente, permitindo analisar a influência do sistema cortical na percepção das aberrações ópticas. Utiliza-se a técnica de Scheiner modificada, onde o ângulo de incidência dos feixes luminosos pode ser modificado de acordo com a resposta do paciente. $\mathrm{O}$ exame demora em média quatro minutos e a colaboração do paciente é fundamental. Esse sistema vem sendo implementado pela Emory Vision Correction Group e ainda não apresenta sistema de excimer laser acoplado (Smirnov, 1961). 
3 Casuística e Métodos 
O presente estudo foi realizado na Eye Clinic Day Hospital - São Paulo - SP, com o apoio do Laboratório de Investigações Médicas (LIM 33) da Clínica Oftalmológica do Hospital das Clínicas da Faculdade de Medicina da Universidade de São Paulo.

\section{1 Ética e informação aos pacientes}

Este estudo teve seu protocolo aprovado pelo Comitê de Ética para Análise de Projetos de Pesquisa - CAPPesq, da Diretoria Clínica do Hospital das Clínicas e da Faculdade de Medicina da Universidade de São Paulo (Anexo).

Os indivíduos incluídos neste estudo receberam, no início, informações detalhadas sob a natureza da investigação e, após os esclarecimentos, assinaram o Termo de Consentimento. 


\subsection{Tipo de estudo}

Realizou-se um estudo clínico e prospectivo com intervenção.

\subsection{Objeto do estudo}

Comparar o desempenho visual de indivíduos portadores de miopia com ou sem astigmatismo, corrigidos com óculos e com três LCG, disponíveis comercialmente.

\subsection{Variáveis}

\subsubsection{Variáveis dependentes:}

3.4.1.1 AV logMAR de alto contraste,

3.4.1.2 sensibilidade ao contraste,

3.4.1.3 aberrações ópticas de alta ordem. 


\subsubsection{Variáveis independentes:}

3.4.2.1 óculos,

3.4.2.2 LCG.

\subsubsection{Variáveis com função descritiva:}

3.4.3.1 sexo,

3.4.3.2 idade,

3.4.3.3 poder dióptrico dos meridianos principais da córnea,

3.4.3.4 erro refrativo (componentes esfera, cilindro e equivalente esférico),

\subsection{População e amostra}

Foram selecionados, consecutivamente, 73 indivíduos, portadores de miopia com ou sem astigmatismo, não usuários de LC, com motivação para o uso de LCG, examinados no Setor de Lentes de Contato da Eye Clinic Day Hospital, no período de $1^{\circ}$ de maio de 2003 a 30 de setembro de 2003. Dentre os casos selecionados, quarenta indivíduos, que preencheram os critérios de inclusão e exclusão, constituíram a amostra do estudo. 


\subsubsection{Critérios de inclusão e exclusão}

\subsubsection{Critérios de inclusão:}

- indivíduos de ambos os sexos, portadores de miopia entre $-1,00 \mathrm{D}$ e -4,50 D, sem ou com astigmatismo até $-0,75 \mathrm{D}$, não usuários de LC, com motivação para uso de LCG, com acuidade visual corrigida com óculos e com as LCG igual ou superior a $20 / 20$;

- capacidade de compreender e seguir as orientações recomendadas de uso e manuseio das LCG;

- assinatura do termo de consentimento.

\subsubsection{Critérios de exclusão:}

- presença de afecção ativa de natureza alérgica, inflamatória ou infecciosa, na superfície ocular e ou de anexos;

- valor do meridiano mais plano da córnea > 46,00 D ou < 40,00 D;

- indivíduos que não puderam ser adaptados com os três tipos de LCG avaliadas;

- desconforto importante no teste de adaptação das LCG;

- recusa em participar do estudo. 


\subsection{Materiais}

As lentes dos óculos foram confeccionadas em policarbonato, com tratamento de superfície anti-risco e anti-reflexo. Os parâmetros das LCG estão no Quadro 3.

Quadro 3. Parâmetros das LCG Acuvue ${ }^{\circledR}$ 2, Biomedics ${ }^{\circledR} 55$ e Focus ${ }^{\circledR}$ 1-2 week

\begin{tabular}{|l|c|c|c|}
\hline Lentes de contato & $\begin{array}{c}\text { Acuvue }{ }^{\circledR} \mathbf{2} \\
\text { (Vistacon J\&J Vision } \\
\text { Care Inc., EUA) }\end{array}$ & $\begin{array}{c}\text { Biomedics }{ }^{\circledR 555} \\
\text { (Ocular Science, } \\
\text { EUA) }\end{array}$ & $\begin{array}{c}\text { Focus } \AA \text { 1-2 week } \\
\text { (Ciba Vision } \\
\text { Corporation, EUA) }\end{array}$ \\
\hline Material & Etafilcon A & Ocufilcon A & Vitifilcon A \\
\hline $\begin{array}{l}\text { Porcentagem de água } \\
\text { (\%) }\end{array}$ & 58 & 55 & 55 \\
\hline $\begin{array}{l}\text { Espessura central LC } \\
\text { de -3,0D (mm) }\end{array}$ & 0,08 & 0,07 & 0,06 \\
\hline Diâmetro (mm) & 14,0 & 14,2 & 14,0 \\
\hline $\begin{array}{l}\mathbf{D K} \\
(\mathbf{c m}\end{array}$ & 28 & 28,2 & 26 \\
\hline
\end{tabular}

\subsection{Procedimentos e técnicas}

Os pacientes foram submetidos, inicialmente, aos seguintes exames: anamnese, exame ocular externo, tonometria com tonômetro a ar (“TOPCON CT 80"), ceratometria, refratometria dinâmica subjetiva, medida da acuidade visual com correção, fundoscopia direta, biomicroscopia do 
segmento anterior e avaliação da relação LC-córnea com as LCG, com lâmpada de fenda "Topcon 7E". Ao final da avaliação, tiveram seus óculos prescritos com lentes de policarbonato, com tratamento de superfície antirisco e anti-reflexo. Posteriormente, retornaram para a adaptação das LCG, quando foram submetidos à medida da $\mathrm{AV} \log \mathrm{MAR}$ de alto contraste e as avaliações de sensibilidade ao contraste e das aberrações de alta ordem. Cada paciente teve um olho escolhido por sorteio para a avaliação do desempenho visual com óculos e LCG (sensibilidade ao contraste) e sem e com LCG (aberrometria). A seqüência de adaptação das três LCG foi definida previamente, por sorteio. Entre cada avaliação de desempenho visual fez-se um intervalo de vinte minutos.

\subsubsection{Técnica de adaptação das lentes de contato gelatinosas}

A avaliação da centralização, movimentação e a sobre-refração foram executadas após vinte minutos da colocação da LCG. A análise da relação lente-córnea foi feita à lâmpada de fenda, solicitando-se ao paciente para olhar na direção da testa do examinador, piscar corretamente e, depois, olhar para os lados. Considerou-se como movimentação adequada da LCG um deslocamento lateral de $1 \mathrm{~mm}$ e vertical de $0,5 \mathrm{~mm}$ a $1 \mathrm{~mm}$ ao movimento ocular. Em seguida, avaliou-se o desempenho visual. 


\subsubsection{Medida da acuidade visual de alto contraste}

Utilizou-se a Tabela de AV do ETDRS (Early Treatment Diabetic Retinopathy Study - Estudo do Tratamento Precoce da Retinopatia Diabética) (Ligth House, New York, EUA), posicionada a uma distância de 6 metros (ou a 20 pés) do sujeito. Usaram-se a mesma sala de exame e a mesma iluminação em todos os exames. A AV logMar de alto contraste foi medida primeiro com os óculos, depois com cada LCG, em ordem definida previamente, por sorteio.

\subsubsection{Medida de sensibilidade ao contraste}

O teste de sensibilidade ao contraste foi realizado com o aparelho CSV-1000 (VectorVision, Ohio, EUA) que verifica freqüências espaciais diferentes $(3,6,12$ e $18 \mathrm{cpd})$ em quatro fileiras (A, B, C, e D). O indivíduo foi posicionado a 2,4 $\mathrm{m}$ (8 pés) da unidade onde se encontrava a tabela, utilizando os óculos e depois cada uma das LCG. Mostrou-se ao paciente o primeiro círculo para que identificasse o padrão. À medida que o paciente "lia" a fila, considerava-se que o padrão encontrava-se no círculo inferior ou superior. Se o padrão não fosse visível, o indivíduo informava que ambos os círculos pareciam iguais, ou seja, com o aspecto de cinza homogêneo. A última resposta que identificava corretamente o padrão determinava o limiar de contraste para cada freqüência espacial testada. 
Os níveis de contraste em cada fila variam de 0,70 a 2,08; 0,91 a 2,$29 ; 0,61$ a 1,99; 0,17 a 1,55 unidades logarítmicas para as freqüências de 3, 6,12 e $18 \mathrm{cpg}$, respectivamente. Os níveis de contraste diminuem de uma forma logarítmica uniforme em passos de 0,15 unidades logarítmicas para os níveis de contraste do 3 ao 8 e 0,17 unidades logarítmicas do 1 ao 3 . A mudança de contraste entre o círculo padrão e o nível 1 é de 0,3 unidades logarítmicas. Marcou-se o nível de contraste da última resposta correta identificada pelo sujeito em cada fileira, e projetou-se a curva de sensibilidade para cada olho em separado.

O instrumento tem uma fonte de retroiluminação que mantém uniforme a superfície do teste. Células fotoelétricas no interior da unidade medem sua iluminação interna e a do ambiente onde é realizado o exame. Um sistema de retroalimentação no circuito calibra a unidade em um nível de 85 ciclos por grau de ângulo visual (cpg) por $\mathrm{mm}^{2}$ (ou candelas $/ \mathrm{mm}^{2}$ ) $\pm 0,1$ unidades logarítmicas de luz na superfície do teste. Essa calibragem é recomendada pelo National Committee on Vision da National Academy of Science.

\subsubsection{Sobre-refração e medida de aberrações ópticas de alta ordem}

A sobre-refração e a medida das aberrações ópticas de alta ordem foram realizadas com o aberrômetro Zywave ${ }^{\circledR}$ (Bausch \& Lomb). Para a obtenção de midríase, instilou-se uma gota de Fenilefrina a 10\%, vinte 
minutos antes do início do exame. Em primeiro lugar, posicionou-se o paciente para que, com a cabeça e o queixo confortavelmente apoiados, pudesse observar 0 alvo. Alinhou-se a pupila nos eixos " $x$ " e " $y$ ", observando-se sua posição no monitor. Após a colocação de foco na íris, com o olho mantido aberto, pressionou-se o comando para iniciar o pré scan. Em seguida, foram pressionados os comandos necessários para dar continuidade ao exame até a obtenção da grade e do centróide, visualizados no monitor, no centro da pupila. Novamente, pressionou-se o comando para calcular as aberrações ópticas. Foi considerado o diâmetro pupilar de $6 \mathrm{~mm}$. O exame foi repetido três vezes. Para efeito de cálculo, foram consideradas as médias dos três exames

\subsection{Instrumentos}

O instrumento de medida incluiu uma ficha clínica elaborada especialmente para o presente estudo.

\subsection{Coleta de dados}

A ficha clínica foi preenchida no momento da realização das avaliações pelo investigador. 


\subsection{Processamento dos dados}

Para o processamento dos dados, foi construído um banco de dados com o software Access do Office 2000. Foram elaborados gráficos e tabelas com o Word e o Excell do Office 2000.

\subsection{Procedimento para análise dos dados}

As análises estatísticas foram realizadas com o auxílio dos programas de computador SPSS 10.0, StatXact 4.0 e GraphPad InStat 3.05.

\subsection{Análise estatística}

\subsubsection{Análise descritiva}

As variáveis contínuas foram analisadas por meio dos valores mensurados (resultados) nos pacientes demandados (unidades amostrais). As variáveis categóricas foram consideradas como fatores de estudo, os quais são Tipo de lente $\left(F_{1}\right)$, Ordem $\left(F_{2}\right)$, Nível de aberração $\left(F_{3}\right)$, Nível de ordem $\left(F_{4}\right)$, Sensibilidade ao contraste $\left(F_{5}\right)$ e Polinômios de Zernike $\left(F_{6}\right)$. As 
variáveis resposta são Sensibilidade (ciclos/grau) para o fator $F_{5}$ e Aberração $(\mu \mathrm{m})$ para os demais fatores. Os valores das respostas foram obtidos nas avaliações feitas junto aos pacientes da amostra. Para as variáveis contínuas, foi feita uma análise descritiva obtendo-se as médias, valores mínimo e máximo e desvio padrão para todos os fatores, em separado e em conjunto.

Para fins de análise estatística, as medidas de acuidade visual foram calculadas em LogMAR. Os valores de sensibilidade ao contraste foram convertidos em unidades de log, conforme tabela fornecida pelo fabricante.

\subsubsection{Análise comparativa}

Para efeito de análise dos resultados da aberração e da sensibilidade, utilizou-se o planejamento experimental fatorial, onde são testados quatro modelos que envolvem a combinação dos cinco fatores do experimento com o fator 1 - Tipo de lente.

A técnica inferencial estatística apresenta os resultados dos testes de hipótese que representam os objetivos da tese. A estatística usada para testar as hipóteses foi a $\mathrm{F}$ de Snedcor (Fisher). Para a significância da rejeição ou não das hipóteses, usou-se o p-valor.

Para a comparação dos grupos dois a dois, foi utilizado o Teste F de Snedcor e na análise de amostras independentes, a prova F de Snedcor (Fisher). 
Todos os testes foram realizados na forma bicaudal e admitindo-se o nível de significância de 5\%. Níveis descritivos (p) inferiores a esse valor foram considerados significantes. Utilizou-se o programa de computador Statistica for Windows. 
4 Resultados 
Os dados demográficos e aspectos clínicos dos indivíduos participantes do estudo estão na Tabela 1.

Tabela 1. Dados demográficos e aspectos clínicos dos indivíduos participantes do estudo. Eye Clinic Day Hospital, São Paulo - 2003

\begin{tabular}{|c|c|c|c|}
\hline Características & & & teste $\mathrm{F}$ \\
\hline Idade (anos) & $24,41(4,49)$ & & \\
\hline \multicolumn{4}{|l|}{ Sexo $(\%)$} \\
\hline $\begin{array}{l}\text { Masculino } \\
\text { Feminino }\end{array}$ & $\begin{array}{l}45,0 \\
55,0\end{array}$ & & \\
\hline \multicolumn{4}{|l|}{ Olho sorteado \% } \\
\hline $\begin{array}{l}\text { Direito } \\
\text { Esquerdo }\end{array}$ & $\begin{array}{l}50,0 \\
50,0\end{array}$ & & \\
\hline \multicolumn{4}{|l|}{ Curvatura corneana (dioptrias) } \\
\hline $\begin{array}{l}\text { Meridiano mais plano (K1) } \\
\text { Meridiano mais curvo (K2) }\end{array}$ & $\begin{array}{l}41,94(1,23) \\
44,21(1,25)\end{array}$ & & \\
\hline Refratometria ocular (média e DP) & Refrator manual & $\begin{array}{l}\text { Aberrômetro } \\
\text { Zywave } ®\end{array}$ & \\
\hline $\begin{array}{l}\text { Esférico } \\
\text { Cilindro } \\
\text { Equivalente esférico }\end{array}$ & $\begin{array}{l}-2,33(0,81) \\
-0,18(0,25) \\
-2,38(0,86)\end{array}$ & $\begin{array}{l}-2,48(1,00) \\
-0,48(0,33) \\
-2,72(1,01)\end{array}$ & $\begin{array}{l}p=0,4556 \\
p=0,0000 \\
p=0,0974\end{array}$ \\
\hline
\end{tabular}

A comparação dos resultados dos exames refratométricos mostrou diferença significativa no componente refrativo cilindro, hipercorrigido com o aberrômetro Zywave®.

Todos os indivíduos apresentaram a AV logMAR de 0,00, com os óculos e com cada uma das três LCG. 
Os resultados da sobre-refração pelo aberrômetro Zywave® com as três LCG, para o diâmetro pupilar de 6 mm, estão na Tabela 2.

Tabela 2. Resultados da sobre-refração em dioptrias pelo Zywave ${ }^{\circledR}$ com as lentes de contato gelatinosas (Acuvue ${ }^{\circledR}$ 2, Biomedics $₫ 55$ e Focus $\AA$ 1-2 week). Eye Clinic Day Hospital, São Paulo - 2003

\begin{tabular}{|c|c|c|c|c|c|}
\hline \multirow{3}{*}{ Componente refrativo } & \multicolumn{5}{|c|}{$\mathrm{N}=40$} \\
\hline & \multicolumn{3}{|c|}{ Lentes de contato } & \multicolumn{2}{|c|}{ teste } \\
\hline & Acuvue $囚 2$ & Biomedics $® 55$ & Focus ${ }^{\circledR} 1-2$ week & $\mathbf{F}$ & $\mathbf{p}$ \\
\hline Esfera & $+0,325 \pm 0,459$ & $+0,287 \pm 0,453$ & $+0,096 \pm 0,371$ & 3,242 & 0,0426 \\
\hline Cilindro & $-0,397 \pm 0,304$ & $-0,383 \pm 0,288$ & $-0,417 \pm 0,270$ & 0,141 & 0,8687 \\
\hline Equivalente Esférico & $+0,126 \pm 0,445$ & $+0,095 \pm 0,435$ & $-0,112 \pm 0,393$ & 3,716 & 0,0272 \\
\hline
\end{tabular}

Houve diferença significativa nos componentes refrativos esfera e equivalente esférico. Os resultados do emprego do teste de contraste envolvendo as médias, duas a duas, mostraram que não houve diferença entre as LCG Acuvue ${ }^{\circledR} 2$ e Biomedics $® 55$ e que houve diferença entre elas e a LCG Focus® 1-2 week.

Os valores de sensibilidade ao contraste e respectivas freqüências espaciais, com os óculos e com as LCG, estão na Tabela 3 e Figura 1. 
Tabela 3. Sensibilidade ao contraste, em unidades logarítmicas e respectivas freqüências espaciais em ciclos por grau, com os óculos e com as lentes de contato gelatinosas (Acuvue ${ }^{\circledR} 2$, Biomedics $\AA 55$ e Focus $₫$ 1-2 week). Eye Clinic Day Hospital, São Paulo - 2003

\begin{tabular}{|c|c|c|c|c|c|c|}
\hline \multirow{3}{*}{$\begin{array}{l}\text { Freqüência } \\
\text { espacial }\end{array}$} & \multirow{3}{*}{ Óculos } & & & & \multicolumn{2}{|c|}{$N=40$} \\
\hline & & \multicolumn{3}{|c|}{ Lentes de Contato } & \multicolumn{2}{|c|}{ teste } \\
\hline & & Acuvue ${ }^{\circledR} 2$ & $\begin{array}{c}\text { Biomedics }{ }^{\circledR} \\
55\end{array}$ & $\begin{array}{c}\text { Focus } \circledast 1-2 \\
\text { week }\end{array}$ & $\mathbf{F}$ & $\mathbf{p}$ \\
\hline Freqüência 3 & $1,904 \pm 0,151$ & $1,911 \pm 0,137$ & $1,915 \pm 0,147$ & $1,904 \pm 0,161$ & 0,054 & 0,9834 \\
\hline Freqüência 6 & $2,151 \pm 0,129$ & $2,174 \pm 0,120$ & $2,174 \pm 0,129$ & $2,151 \pm 0,160$ & 0,364 & 0,7790 \\
\hline Freqüência 12 & $1,814 \pm 0,134$ & $1,836 \pm 0,120$ & $1,814 \pm 0,154$ & $1,807 \pm 0,145$ & 0,312 & 0,7951 \\
\hline Freqüência 18 & $1,344 \pm 0,139$ & $1,359 \pm 0,148$ & $1,359 \pm 0,140$ & $1,329 \pm 0,140$ & 0,409 & 0,7470 \\
\hline
\end{tabular}

Os valores de sensibilidade ao contraste não foram influenciados pela correção dos erros refrativos com os óculos ou com as LCG.

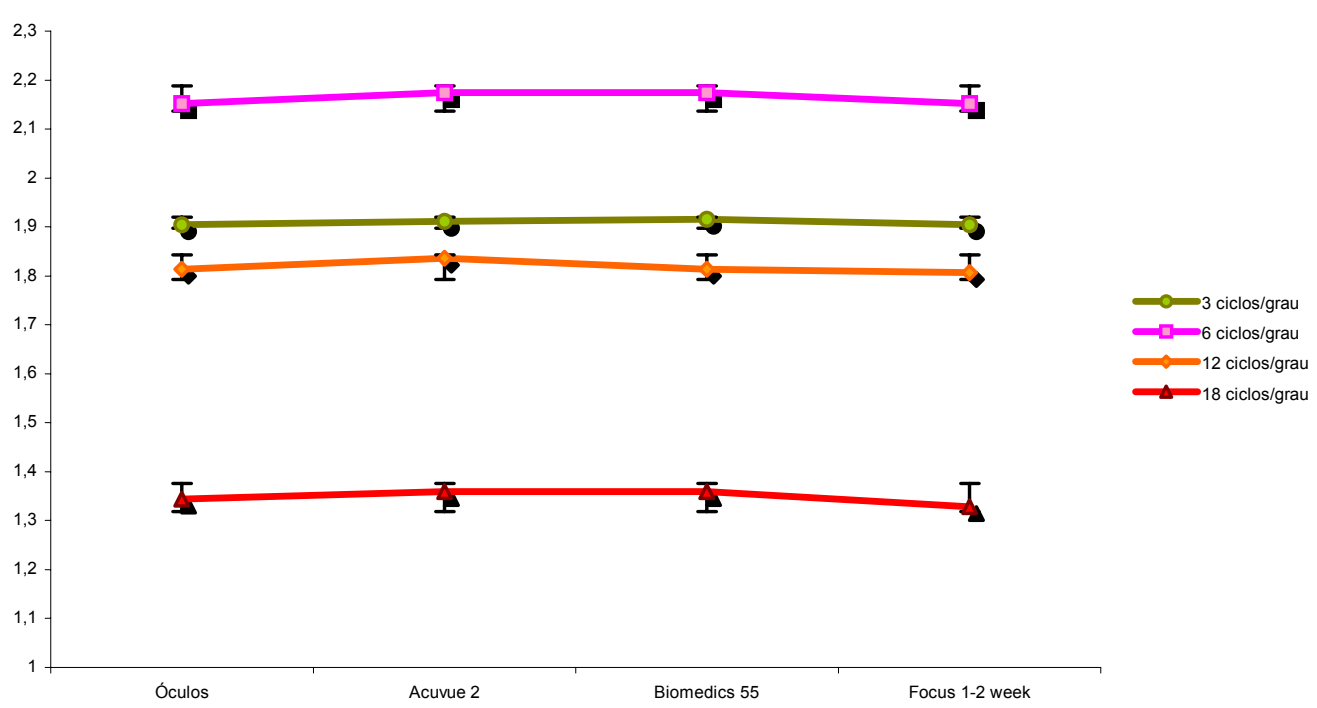

Figura 1. Representação gráfica dos valores de sensibilidade ao contraste em unidades logarítmicas nas freqüências 3, 6, 12 e 18 ciclos por grau com os óculos e com as lentes de contato gelatinosas (Acuvue ${ }^{\circledR}$ 2, Biomedics $\circledR 55$ e Focus ${ }^{\circledR}$ 1-2 week). Eye Clinic Day Hospital, São Paulo - 2003 
Os resultados das aberrações de alta ordem representadas pelos polinômios de Zernike, para o diâmetro pupilar de $6 \mathrm{~mm}$, encontram-se na tabela 4.

Tabela 4. Aberrações de alta ordem representadas pelos polinômios de Zernike em RMS em micrômetros sem e com as lentes de contato gelatinosas (Acuvue $₫$ 2, Biomedics $\AA 55$ e Focus $₫$ 1-2 week). Eye Clinic Day Hospital, São Paulo - 2003

\begin{tabular}{|c|c|c|c|c|c|c|}
\hline \multirow{3}{*}{$\begin{array}{l}\text { Polinômios } \\
\text { de Zernike }\end{array}$} & \multirow[b]{3}{*}{ Sem LC } & \multicolumn{5}{|c|}{$\mathrm{N}=40$} \\
\hline & & \multicolumn{3}{|c|}{ Lentes de Contato } & \multicolumn{2}{|c|}{ teste } \\
\hline & & Acuvue ${ }^{\circledR} 2$ & $\begin{array}{c}\text { Biomedics } ₫ \\
55 \\
\end{array}$ & $\begin{array}{c}\text { Focus } ₫ 1-2 \\
\text { week }\end{array}$ & $\mathbf{F}$ & p \\
\hline Z311 & $0,142 \pm 0,071$ & $0,180 \pm 0,103$ & $0,150 \pm 0,119$ & $0,177 \pm 0,102$ & 1,44 & 0,2327 \\
\hline Z310 & $0,088 \pm 0,064$ & $0,083 \pm 0,071$ & $0,099 \pm 0,094$ & $0,0998 \pm 0,071$ & 0,41 & 0,7488 \\
\hline Z331 & $0,146 \pm 0,113$ & $0,079 \pm 0,055$ & $0,074 \pm 0,044$ & $0,169 \pm 0,094$ & 13,65 & 0,0000 \\
\hline Z330 & $0,128 \pm 0,127$ & $0,073 \pm 0,051$ & $0,087 \pm 0,075$ & $0,093 \pm 0,067$ & 2,94 & 0,0349 \\
\hline Z400 & $0,083 \pm 0,059$ & $0,1007 \pm 0,073$ & $0,110 \pm 0,055$ & $0,0807 \pm 0,060$ & 2,07 & 0,1069 \\
\hline Z420 & $0,039 \pm 0,033$ & $0,0446 \pm 0,029$ & $0,029 \pm 0,026$ & $0,0388 \pm 0,032$ & 1,75 & 0,1581 \\
\hline Z421 & $0,023 \pm 0,021$ & $0,0287 \pm 0,032$ & $0,029 \pm 0,035$ & $0,0352 \pm 0,026$ & 1,36 & 0,3364 \\
\hline Z440 & $0,052 \pm 0,033$ & $0,0376 \pm 0,026$ & $0,0322 \pm 0,030$ & $0,0411 \pm 0,035$ & 2,94 & 0,0349 \\
\hline Z441 & $0,0362 \pm 0,022$ & $0,0385 \pm 0,026$ & $0,0344 \pm 0,026$ & $0,0483 \pm 0,027$ & 2,36 & 0,0732 \\
\hline Z510 & $0,023 \pm 0,020$ & $0,02037 \pm 0,018$ & $0,0204 \pm 0,015$ & $0,0180 \pm 0,014$ & 0,57 & 0,6336 \\
\hline Z511 & $0,0227 \pm 0,018$ & $0,0311 \pm 0,022$ & $0,0185 \pm 0,017$ & $0,0280 \pm 0,023$ & 3,04 & 0,0309 \\
\hline Z530 & $0,0180 \pm 0,014$ & $0,0142 \pm 0,011$ & $0,01405 \pm 0,012$ & $0,0171 \pm 0,022$ & 0,70 & 0,5542 \\
\hline Z531 & $0,0196 \pm 0,013$ & $0,0179 \pm 0,014$ & $0,0192 \pm 0,013$ & $0,0231 \pm 0,018$ & 0,92 & 0,4325 \\
\hline Z550 & $0,0261 \pm 0,018$ & $0,0249 \pm 0,016$ & $0,0195 \pm 0,016$ & $0,0262 \pm 0,019$ & 1,35 & 0,2613 \\
\hline Z551 & $0,0191 \pm 0,014$ & $0,0162 \pm 0,011$ & $0,0267 \pm 0,016$ & $0,0229 \pm 0,023$ & 32,07 & 0,0000 \\
\hline
\end{tabular}

Houve diferença significativa nos polinômios Z331, Z330, Z440, Z511 e Z551. Os resultados do emprego do teste de contraste envolvendo as médias, duas a duas, em ordem decrescente de RMS, mostraram que: 
- polinômio Z331: (Acuvue® 2 = Biomedics ${ }^{\circledR}$ 55) \# sem LC \# Focus ${ }^{\circledR} 1-2$ week;

- polinômios Z330 e Z440: $($ Acuvue $® 2$ = Biomedics $® 55$ = Focus $\AA$ 1-2 week) \# sem LC;

- polinômio Z511: (Biomedics® 55= sem LC) \# (Focus® 1-2 week = Acuvue® 2);

- polinômio Z551: (Acuvue® 2 = sem LC) \# (Biomedics® 55 = Focus® 1-2 week).

Os valores das aberrações de alta ordem em RMS, para o diâmetro pupilar de $6 \mathrm{~mm}$, estão na Tabela 5 .

Tabela 5. Aberrações de alta ordem em RMS em micrômetros, sem e com as lentes de contato gelatinosas (Acuvue ${ }^{\circledR} 2$, Biomedics $\circledR 55$ e Focus ${ }^{\circledR}$ 1-2 week). Eye Clinic Day Hospital, São Paulo - 2003

\begin{tabular}{|c|c|c|c|c|c|c|}
\hline \multirow{3}{*}{ ORDEM } & \multirow{3}{*}{ Sem LC } & & & & \multicolumn{2}{|c|}{$N=40$} \\
\hline & & \multicolumn{3}{|c|}{ Lentes de contato } & \multicolumn{2}{|c|}{ Teste } \\
\hline & & Acuvue ${ }^{\circledR} 2$ & $\begin{array}{c}\text { Biomedics }{ }^{\circledR} \\
55\end{array}$ & $\begin{array}{l}\text { Focus } \AA \text { 1-2 } \\
\text { week }\end{array}$ & $\begin{array}{c}F \text { de } \\
\text { Snedcor }\end{array}$ & p-valor \\
\hline Terceira & $0,302 \pm 0,168$ & $0,254 \pm 0,084$ & $0,243 \pm 0,060$ & $0,306 \pm 0,020$ & 3,637 & 0,029 \\
\hline Quarta & $0,129 \pm 0,136$ & $0,144 \pm 0,129$ & $0,139 \pm 0,056$ & $0,134 \pm 0,018$ & 0,295 & 0,745 \\
\hline Quinta & $0,063 \pm 0,130$ & $0,062 \pm 0,116$ & $0,058 \pm 0,049$ & $0,066 \pm 0,034$ & 1,038 & 0,357 \\
\hline
\end{tabular}

Houve diferença significativa na aberração de terceira ordem. Os resultados do emprego do teste de contraste envolvendo as médias, duas a duas, em ordem decrescente de RMS, mostraram que:

- $\quad($ Acuvue $₫ 2$ = Biomedics $\circledR 55) \#($ Focus $® 1-2$ week = sem LC $)$. 
Na Figura 2, está a representação gráfica dos valores de RMS da aberração de terceira ordem.

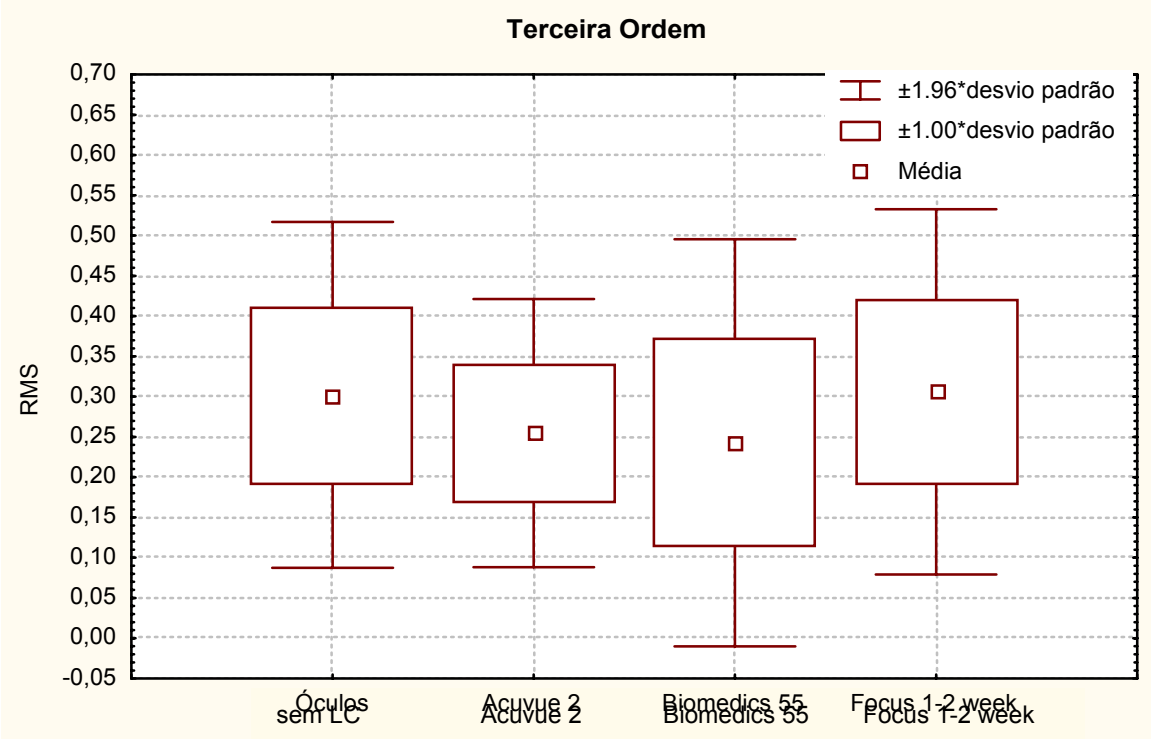

Figura 2. Representação gráfica dos valores de RMS em micrômetros da aberração de terceira ordem sem e com as lentes de contato gelatinosas (Acuvue $\AA$ 2, Biomedics $\AA 55$ e Focus $\AA$ 1-2 week). Eye Clinic Day Hospital, São Paulo - 2003 
Os valores dos níveis das aberrações de alta ordem sem e com as LCG, para o diâmetro pupilar de 6 mm, estão na Tabela 6.

Tabela 6. Níveis de aberrações ópticas em RMS em micrômetros, sem e com as lentes de contato gelatinosas (Acuvue ${ }^{\circledR}$ 2, Biomedics $®$ 55 e Focus ${ }^{\circledR}$ 1-2 week). Eye Clinic Day Hospital, São Paulo 2003

\begin{tabular}{|c|c|c|c|c|c|c|}
\hline \multirow[b]{3}{*}{ Nível de Aberração } & \multirow[b]{3}{*}{ Sem LC } & \multicolumn{5}{|c|}{$N=40$} \\
\hline & & \multicolumn{3}{|c|}{ Lentes de Contato } & \multicolumn{2}{|c|}{ Teste } \\
\hline & & Acuvue ${ }^{\circledR} 2$ & $\begin{array}{c}\text { Biomedics }{ }^{\circledR} \\
55\end{array}$ & $\begin{array}{c}\text { Focus }{ }^{\circledR} 1-2 \\
\text { week }\end{array}$ & $\mathbf{F}$ & $\mathbf{p}$ \\
\hline Coma & $0,177 \pm 0.075$ & $0,210 \pm 0.104$ & $0,190 \pm 0.138$ & $0,213 \pm 0.104$ & 0,634 & 0,457 \\
\hline Trifoil & $0,221 \pm 0.133$ & $0,117 \pm 0.060$ & $0,131 \pm 0.057$ & $0,204 \pm 0.094$ & 16,620 & 0,000 \\
\hline Aberração esférica & $0,083 \pm 0.059$ & $0,101 \pm 0.073$ & $0,110 \pm 0.055$ & $0,081 \pm 0.060$ & 2,276 & 0,107 \\
\hline Astigmatismo secundário & $0,050 \pm 0.034$ & $0,058 \pm 0.036$ & $0,047 \pm 0.037$ & $0,058 \pm 0.034$ & 1,286 & 0,280 \\
\hline Tetrafoil & $0,067 \pm 0.032$ & $0,059 \pm 0.027$ & $0,053 \pm 0.030$ & $0,070 \pm 0.034$ & 2,997 & 0,054 \\
\hline Coma secundário & $0,036 \pm 0.022$ & $0,040 \pm 0.024$ & $0,031 \pm 0.018$ & $0,037 \pm 0.022$ & 2,069 & 0,131 \\
\hline Trifoil secundário & $0,029 \pm 0.014$ & $0,025 \pm 0.013$ & $0,026 \pm 0.014$ & $0,031 \pm 0.025$ & 1,143 & 0,322 \\
\hline Pentafoil & $0,035 \pm 0.017$ & $0,032 \pm 0.015$ & $0,036 \pm 0.017$ & $0,039 \pm 0.025$ & 1,179 & 0,311 \\
\hline
\end{tabular}

Houve diferença significativa no nível de aberração trifoil. Os resultados do emprego do teste de contraste envolvendo as médias, duas a duas, em ordem decrescente de RMS, mostraram que:

- $\quad($ Acuvue® 2 = Biomedics® 55) \# Focus $® 1-2$ week \# sem LC. 
Na Figura 3, está a representação gráfica dos valores de RMS da aberração trifoil.

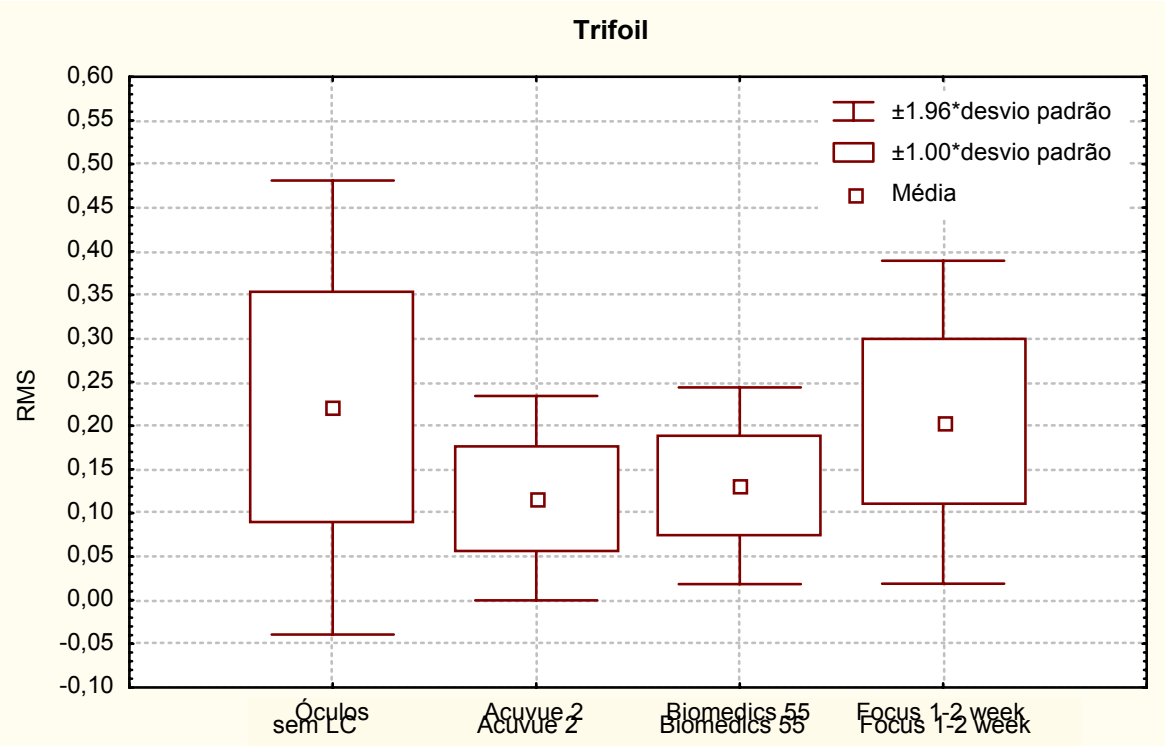

Figura 3. Representação gráfica dos valores de RMS em micrômetros da aberração trifoil sem e com as lentes de contato gelatinosas (Acuvue $₫$ 2, Biomedics $₫ 55$ e Focus $₫$ 1-2 week). Eye Clinic Day Hospital, São Paulo - 2003 
5 Discussão 
A despeito da qualidade óptica das LC ter melhorado, alguns indivíduos queixam-se da visão corrigida com LC, embora apresentem AVcc de 20/20 (Wachler et al., 1999). Vários investigadores analisaram o desempenho visual com LC e com óculos e verificaram diminuição de sensibilidade ao contraste com as LC em comparação com os óculos (Applegate, Massof, 1975; Wo, Hess, 1979; Mitra, Lamberts, 1981; Kirkpatrik, Roggenkamp, 1985; Cox, Holden, 1990). As LCG são amplamente utilizadas, mas muito pouco se conhece do desempenho visual com LCG de diferentes fabricantes. Este estudo foi desenhado para comparar o desempenho visual de três LCG, disponíveis comercialmente, com o de óculos, utilizando-se na avaliação, AV logMAR de alto contraste, sensibilidade ao contraste e análise de frentes de onda.

A comparação dos resultados dos exames refratométricos, subjetivo com retinoscopia e refrator manual e objetivo com o aberrômetro Zywave, mostrou diferença significativa no componente refrativo cilindro, hipercorrido com o aberrômetro Zywave® (Tabela 1). O emprego do aberrômetro para determinar o estado refrativo do olho apresenta menor acurácia que os refratores automáticos convencionais, sendo necessárias várias medidas e a utilização de suas médias (Hament, 2002; Schimitzek, Wasemann, 2002; Mirshahi et al., 2003). Neste estudo, utilizaram-se as médias de três medidas. 
Os resultados da sobre-refração objetiva pelo aberrômetro Zywave® com as LCG evidenciaram diferenças significativas nos componentes refrativos esfera e equivalente esférico (Tabela 2). As LCG Acuvue® 2 e Biomedics ${ }^{\circledR} 55$ não apresentaram diferenças nos componentes esfera e equivalente esférico, que se mostraram hipercorrigidos em relação a LCG Focus ${ }^{\circledR}$ 1-2 week. Entre as três LCG, não houve diferença significativa no componente cilindro. Esses resultados mostram que as LCG não corrigiram igualmente os erros refrativos. Thibos et al. (2002) mostraram que, em olhos bem corrigidos com LC, as aberrações de alta ordem equivalem a cerca de 0,25 D. Para esses autores deve-se procurar corrigir na LC as pequenas frações dos erros refrativos esferoclíndricos para se conseguir reduzir as aberrações de alta ordem.

Não houve diferença na AV logMAR de alto contraste entre os óculos e as três LCG. O critério de inclusão no estudo selecionou indivíduos portadores de miopia e astigmatismo até $-0,75$ D com AV cc de 20/20 (Snellen). Em investigações anteriores, Cox e Holden (1990) e Wachler et al. (1999) mostraram que a correção de erros refrativos com óculos ou com LCG não apresentava diferenças de desempenho visual, quando avaliado pela AV logMar cc de alto contraste. Para esses autores, o desempenho visual deveria ser avaliado pela sensibilidade ao contraste. A sensibilidade ao contraste é uma medida mais sensível da função visual que a AV logMAR de alto contraste, não somente na avaliação com LC, mas também em vários estados de doença ocular (Wolkstein et al., 1980, Sekuler et al., 1986; Wachler et al., 1999). 
Em geral, a AV é mensurada por meio de testes de acuidade visual que determinam o menor detalhe que pode ser visualizado usando letras ou símbolos de cor preta contra um fundo branco com quase $100 \%$ de contraste. Os testes de sensibilidade ao contraste espacial medem a capacidade de detectar diferenças entre áreas de diferentes luminâncias, situadas lado a lado no espaço, possibilitando uma avaliação mais precisa da AV espacial do indivíduo do que os testes de mensuração de AV com os optotipos (Wolkstein et al., 1980; Sekuler et al., 1986; Wachler et al., 1999). No entanto, neste estudo, a avaliação de sensibilidade ao contraste não identificou diferenças entre os óculos e as três LCG (Tabela 3 e Figura 1). Tais resultados diferem dos achados de Cox e Holden (1990) que encontraram redução de sensibilidade ao contraste com LCG em comparação com os óculos. Diferem, também, dos achados de Wachler et al. (1999) que encontraram redução significativa de sensibilidade ao contraste, na freqüência de 12 ciclos por grau, quando compararam a LC Cibasoft com os óculos. A redução de sensibilidade ao contraste em freqüências mais baixas foi igualmente observada com LCG com baixo conteúdo de água (Grey, 1986-a) e com maior espessura central (Grey, 1986-b). Embora, neste estudo, as LCG sejam de fabricantes e de materiais diferentes (Quadro 3), não foi possível, por meio da medida de sensibilidade ao contraste, identificar diferenças no desempenho visual entre elas e os óculos (Tabela 3 e Figura 1).

O grande diferencial desta pesquisa foi comparar o desempenho visual sem e com as LCG considerando as aberrações ópticas de alta 
ordem, que não são passíveis de correção com óculos. Na Tabela 4, as aberrações ópticas de alta ordem estão representadas pelos polinômios de Zernike, que são uma maneira de se caracterizar e quantificar superfícies permitindo a sua comparação. Em relação aos coeficientes de terceira ordem, ocorreu diferença significativa nos polinômios Z330 e Z331 que representam, conjuntamente, a aberração trifoil. Em relação ao coeficiente Z330 não houve diferença significativa no desempenho visual entre as três LCG, que foi superior ao observado sem LC. Em relação ao polinômio Z331, não houve diferença significativa no desempenho visual entre as LCG Acuvue $\circledast 2$ e Biomedics $® 55$, que foi superior àquele sem LC e com a LCG Focus $₫$ 1-2 week. Em relação ao polinômio Z440, não houve diferença significativa no desempenho visual entre as três LCG, o qual foi superior ao sem LC. Em relação aos coeficientes de quinta ordem, considerando-se o polinômio Z511, não houve diferença significativa no desempenho visual entre as LCG Biomedics ${ }^{\circledR} 55$ e sem LC, que foi superior ao observado com as LCG Focus ${ }^{\circledR}$ 1-2 week e Acuvue ${ }^{\circledR} 2$ e, em relação ao polinômio Z551, não houve diferença significativa no desempenho visual entre as LCG Acuvue ${ }^{\circledR} 2$ e sem LC, que foi superior ao observado com as LCG Biomedics $₫ 55$ e Focus $® 1-2$ week.

Os resultados das aberrações de alta ordem estão na Tabela 5 e na Figura 2. Houve diferença significativa na aberração de terceira ordem sem e com as LCG. Não houve diferença entre o desempenho visual com as LCG Acuvue $₫ 2$ e Biomedics $₫ 55$, o qual foi superior ao observado com a LCG Focus $®$ 1-2 week e sem LCG. Observou-se que, em termos individuais, a 
maior média absoluta dos valores de RMS foi a dos níveis de aberração coma, trifoil e tetrafoil (Tabela 6). Esta ordem de distribuição dos resultados foi concordante com a encontrada por Wang e Koch (2003), em avaliação realizada em 532 olhos de 306 indivíduos normais. As aberrações de alta ordem representam o agrupamento das aberrações de coma, trifoil, aberração esférica, astigmatismo secundário, tetrafoil, coma secundário, trifoil secundário e pentafoil. A análise comparativa da distribuição dessas aberrações entre os olhos sem LC e com LCG mostrou diferença estatisticamente significativa no nível trifoil (Tabela 6 e Figura 2). Em relação ao componente trifoil, o teste de contraste comparando as médias, duas a duas, mostrou maior nível de aberração sem LCG, e em ordem decrescente de RMS, LCG Focus ${ }^{\circledR}$ 1-2 week, Biomedics ${ }^{\circledR} 55$ e Acuvue ${ }^{\circledR}$ 2. Segundo Applegate et al. (2003), níveis diferentes de aberração com o mesmo valor de RMS influenciam de forma distinta o desempenho visual. Para esses autores, os níveis de aberração que se situam mais ao centro da pirâmide de Zernike afetam mais a qualidade visual do que aqueles que se situam mais próximos da periferia da pirâmide. Do mesmo modo, deformações nas frentes de onda mais próximas do centro da pupila apresentam maior tendência para influenciar negativamente a qualidade visual (Applegate et al., 2003). Ainda segundo esses autores, em avaliação da qualidade da visão por meio de tabelas "aberradas" geradas por computação, o nível de aberração "coma" diminuiu mais significativamente a visão do que o trifoil. Para Wachler et al. (1999), a medida de AV não reflete o desempenho visual, sendo comum indivíduos com AVcc de 20/20 reclamarem dessa 
visão. Para Applegate et al. (2003), as correções ópticas guiadas por frentes de onda deveriam ser capazes de corrigir dentro de 1/8 D do erro refrativo, tanto em esfera como em cilindro, sem induzir novas aberrações de alta ordem. Ressaltam, também, que novos testes clínicos têm de ser desenvolvidos com a capacidade de demonstrar acuradamente o ganho ou a perda em sensibilidade ao contraste provocada por pequenas alterações nos níveis de aberração óptica ocular.

As aberrações ópticas, neste estudo, foram medidas com o aberrômetro Zywave® que utiliza o sensor de Hartmann-Shack, o qual mede as aberrações oculares totais. Outros videoceratoscópios computadorizados podem medir a forma corneana, desenhar mapas de elevação e realizar a decomposição polinomial que é utilizada para determinar as aberrações corneanas (Oshika et al., 1999; Wang et al., 2002). O conhecimento das aberrações de frentes de onda corneana e lenticular é essencial para o entendimento da natureza de cada aberração e para sua correção. Esse conhecimento é fundamental para se obter, de forma personalizada, LC e lentes intra-oculares para a correção de aberrações ópticas presentes em pacientes candidatos à lensectomia refrativa ou à cirurgia de catarata (Wang et al. 2002). Na prática, existem inúmeras limitações para a correção com êxito de aberrações de alta ordem com LC. Para melhorar o desempenho visual com LC, é necessário reduzir as aberrações de baixa ordem corrigindo-se os erros refrativos de maneira mais acurada que o realizado hoje, na rotina da prática clínica. Thibos et al. (2002) mostraram que, em olhos bem corrigidos com LC, as aberrações de alta ordem equivalem a 
cerca de 0,25 D. Para esses autores, deve-se procurar corrigir na LC as pequenas frações dos erros refrativos esferoclíndricos a fim de se conseguir reduzir as aberrações de alta ordem. Para Guirao et al. (2001) e Guirao et al. (2002), a centralização, movimentação e rotação das LC dificultam a correção de aberrações de alta ordem. Para a correção de aberrações de alta ordem com LC, são necessários alinhamento e estabilidade rotacional mais acurados que os requeridos para se obter bom desempenho visual com LC tórica. Qualquer movimento da LC durante o piscar ou rotação ocular diminui a efetividade da correção óptica. Com os desenhos atuais das LC, ainda não é possível conseguir a estabilidade necessária para a correção efetiva de aberrações de alta ordem (Guirao et al., 2001; Guirao et al., 2002). Para He et al. (2000), há muito que aprender, neste caminho, sobre as aberrações ópticas oculares. Primeiro porque, sendo o bulbo ocular um sistema biológico, está sofrendo mudanças - fisiológicas ou não - todo o tempo. Modificações das aberrações ópticas ocorrem com a acomodação, outra fonte de variabilidade biológica. Segundo, porque as medidas dos níveis de aberração são influenciadas por fatores variáveis, como o alinhamento do aberrômetro durante o exame ou até por pequenos erros de fixação.

A qualidade óptica da imagem retínica é degradada por dispersão, difração, aberração cromática e aberração monocromática (Collins et al., 1995). A dispersão é pouco relevante em olhos sadios de indivíduos jovens, como os que foram selecionados para a amostra deste estudo. A difração depende do diâmetro da pupila e deve ser considerada em pupilas menores 
que $1 \mathrm{~mm}$ (Katz, 1982; Collins et al., 1995). Portanto, as principais causas de embaçamento visual em olhos sadios de indivíduos jovens são as aberrações cromáticas e monocromáticas (Collins et al., 1995). A aberração cromática decorre do fato de cada comprimento de onda da luz ter índice refrativo distinto e, por isso, cada cor é refratada diferentemente quando comparada com outra. A diferença cromática total no espectro visual inteiro é de quase 2,00D; sendo assim, tem efeitos importantes na degradação da imagem retínica e, conseqüentemente, na $\mathrm{AV}$ (Collins et al., 1995). A aberração monocromática decorre do fato de que lentes reais (lentes dos óculos, LC ou componentes refrativos do bulbo ocular) modificam uma frente de onda plana incidente de modo que os raios paracentrais acabam alterados de maneira diferente em comparação com os periféricos, causando a não coincidência com um ponto focal único (Collins et al., 1995). Para Applegate et al. (2003), os erros de frentes de onda constituem o principal fator para a degradação da imagem retínica em olhos sadios com pupilas maiores do que $3,0 \mathrm{~mm}$ e com mínima alteração de transparência dos meios ópticos (ausência de catarata).

Por que a avaliação da AV LogMAR de alto contraste e da sensibilidade ao contraste não identificaram diferenças no desempenho visual sem e com as LCG? Porque, segundo Applegate et al. (2003), os níveis de aberração que se situam próximo da periferia da pirâmide de Zernike afetam pouco a qualidade visual. Neste estudo, não houve diferença significativa em relação aos níveis de aberração coma e esférica que se situam mais ao centro da pirâmide. No entanto, é importante enfatizar que 
cada nível de aberração tem um diferente impacto no desempenho visual, se avaliado em baixo ou em alto contraste. Um nível de aberração pode estar sendo usado para balancear outro e melhorar a modulação da função de transferência (MTF), bem como a qualidade da imagem retínica (Charman, 1991; Thibos et al., 2002; Applegate et al., 2003). Applegate et al. (2003) mostraram que, quando combinados, os níveis de aberrações de alta ordem podem aumentar ou diminuir o desempenho visual. Esses autores notaram que, quando combinados, distintos níveis de aberração podem interagir para melhorar a qualidade visual. Para eles, a combinação de aberração esférica com defocus afeta menos o desempenho visual que seus efeitos individuais.

A escolha dos indivíduos participantes do estudo é fundamental para garantir que os resultados representem, fielmente, o que ocorre na população de interesse (Hulley et al., 2003). Nesta investigação, selecionouse uma amostra de pessoas viáveis de serem estudadas, em termos de tempo e custos financeiros, e que fosse, ao mesmo tempo, grande o suficiente para controlar o erro amostral na generalização dos achados e representativa para controlar o erro sistemático dessas inferências. Para o cálculo do erro amostral para a amostra de quarenta indivíduos, utilizou-se a técnica de amostragem aleatória para população finita com variáveis contínuas (sensibilidade ao contraste e aberrações de alta ordem). Os desvios-padrão dessas duas variáveis foram, respectivamente, 0,603 e 0,326. Os desvios-padrão foram calculados para a amostra submetida a medidas repetidas. O valor calculado do erro amostral foi de 0,03. As medidas de sensibilidade ao contraste e de aberrações ópticas de alta 
ordem variam grandemente entre indivíduos e aumentam com a idade (Porter et al., 2001; Castejón-Mochón et al., 2002; Wang et al., 2002). Neste estudo, portanto, todos os indivíduos foram submetidos a medidas repetidas de AV logMAR de alto contraste, sensibilidade ao contraste e de aberrações de alta ordem, evitando-se, assim, a composição de grupos, eliminando-se a variação interindivíduos e, dessa forma, otimizando-se o tamanho da amostra. Para controlar o erro sistemático decorrente de inferências, utilizouse um modelo fatorial para identificar os efeitos da correção dos erros refrativos com óculos e sem e com as três LCG sobre as respostas de sensibilidade ao contraste e de aberrações de alta de ordem. A generalização dos resultados fica, portanto, restrita a uma população de indivíduos com características semelhantes às daqueles que compuseram a amostra, que serviu de base para este estudo. 
6 Conclusões 
Este estudo foi realizado para comparar o desempenho visual de indivíduos portadores de miopia com ou sem astigmatismo, corrigidos com óculos e com as LCG Acuvue® 2, Biomedics ${ }^{\circledR} 55$ e Focus ${ }^{\circledR}$ 1-2 week. Nas condições propostas para este estudo, conclui-se que:

1. pela sobre-refração objetiva realizada pelo aberrômetro Zywave®, em relação aos componentes refrativos:

- não houve diferença significativa entre as LCG Acuvue® 2 e Biomedics $® 55$;

- houve diferença significativa nos componentes esfera e equivalente esférico, entre a LCG Focus® 1-2 week e as LCG Acuvue® 2 e Biomedics $® 55$.

2. pela medida da AV LogMAR não foram detectadas diferenças significativas no desempenho visual com os óculos e com as três LCG.

3. pela avaliação de sensibilidade ao contraste não foram detectadas diferenças significativas no desempenho visual com óculos e com as três LCG. 
4. na análise de frentes de onda:

- houve diferença significativa na aberração de terceira ordem com e sem as LCG. Não houve diferença significativa no desempenho visual entre as LCG Acuvue® 2 e Biomedics $® 55$, que foi superior ao observado com a LCG Focus® 1-2 week;

- em relação ao polinômio de Zernike Z331, não houve diferença significativa no desempenho visual entre as LCG Acuvue® 2 e Biomedics $® 55$, que foi superior ao sem LC e com a LCG Focus $®$ 1-2 week;

- em relação aos polinômios de Zernike Z330 e Z440, não houve diferença significativa entre as três LCG, que foi superior ao sem LC;

- em relação ao polinômio Z511, não houve diferença significativa no desempenho visual entre as LCG Biomedics ${ }^{\circ} 55$ e sem LC, que foi superior ao observado com as LCG Focus® 1-2 week e Acuvue ${ }^{2}$ 2;

- em relação ao polinômio Z551, não houve diferença significativa no desempenho visual entre as LCG Acuvue® 2 e sem LC, que foi 
superior ao observado com as LCG Biomedics ${ }^{\circledR} 55$ e Focus® 1-2 week;

- em relação ao nível trifoil de aberração de terceira ordem, representado pelo agrupamento dos polinômios Z330 e Z331, não houve diferença significativa entre as LCG Acuvue® 2 e Biomedics $® 55$, que foi superior ao observado com a LCG Focus $®$ 1-2 week e sem LC.

5. na avaliação do desempenho visual com óculos e LCG, a análise de frentes de onda mostrou ser uma medida mais sensível da função visual que a AVLogMAR de alto contraste e a sensibilidade ao contraste.

6. o modelo de avaliação de desempenho visual utilizado nesta investigação serve de modelo para pesquisas semelhantes. 
7 Anexo 


\title{
ANEXO - Carta do Comitê de Ética
}

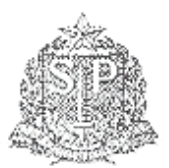

\author{
HOSPITAL DAS CLINICAS

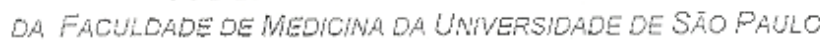 \\ CAIXA POSTAL, 8091 - SÄO PAULO-BRASIL \\ DIRETORIA CLÍNICA
}

Comissão de Ética para Análise de Projetos de Pesquisa

\section{APROVACÃO}

A Comissão de Ética para Análise de Projetos de Pesquisa - CAPPesq da Diretoria Clínica do Hospital das Clínicas e da Faculdade de Medicina da Universidade de São Paulo, em sessão de 14.05.03, APROVOU o Protocolo de Pesquisa $n^{\circ}$ 029/03, intitulado: "Comparação das aberraçöes ópticas e da sensibilidade ao contraste em diferentes tipos de lentes de contato" apresentado pelo Departamento de OFTALMOLOGIA E OTORRINOLARINGOLOGIA, bem como o Termo de Consentimento Livre e Esclarecido.

Pesquisador(a) Responsável: PROF. DR. NEWTON KARA JOSÉ

Pesquisador(a) Executante: DR. BRENO BARTH AMARAL DE ANDRADE

CAPPesq, 14 de Maio de 2003.

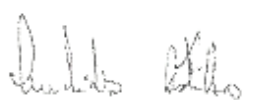

PROF. DR. EUCLIDES AYRES DE CASTILHO

Presidente da Comissão Ética para Análise

de Projetos de Pesquiso

OBSERVAÇÃO: Cabe aO pesquisador elaborar e apresentar à CAPPesq, os réatórios parciais e final sobre a pesquisa (Resoluçäo do Conseltho Nacional de Saúde $n^{\circ} 196$, de 10.10.1996, inciso IX.2, letro " $\mathrm{C}$ ") 


\section{Referências ${ }^{1}$}

${ }^{1}$ De acordo com:

Adaptado de International Committee of Medical Journals Editors (Vancouver).

Universidade de São Paulo. Faculdade de Medicina. Serviço de Biblioteca e Documentação. Guia de apresentação de dissertações, teses e monografias da FMUSP. Elaborado por Anneliese Carneiro da Cunha, Maria Julia A.L. Freddi, Maria F. Crestana, Marinalva de S. Aragão, Suely C. Cardoso, Valéria Vilhena. São Paulo: Serviço de Biblioteca e Documentação, 2004.

Abreviaturas dos títulos dos periódicos de acordo com List of Journals Indexed in Index Medicus. 
Aizawa D, Shimizu K, Komatsu M, Ito M, Suzuki M, Ohno K, Uozato H. Clinical outcomes of wavefront-guided laser in situ keratomileusis: 6-month follow-up. J Cataract Refract Surg. 2003;29:1507-13.

Alves MR. Considerações anatômicas e fisiológicas para a adaptação de lentes de contato. In: Coral-Ghanem C, Kara-José N, editores. Lentes de Contato. Rio de Janeiro: Cultura Médica; 2003. p.55-67.

Applegate RA, Marsak JD, Ramos R, Sarver EJ. Interaction between aberrations to improve or reduce visual performance. J Cataract Refract Surg. 2003;29:1487-95.

Applegate RA, Massof RW. Changes in the contrast sensitivity functions induced by contact lenses wear. Am J Optom Phisiol. Opt. 1975;52:840-6.

Argento C, Cosentino MJ. Customized ablation for asymmetrical corneal astigmatism. J Cataract Refract Surg. 2001;27:545-6.

Atchinson DA. Spectacle lens design: development and present state. Aust J Optom. 1984;67:97-113.

Atchinson DA. Modern optical designs assessment and spectacle lenses. Optica Acta. 1985;32:607-34.

Bailey IL. Visual acuity. In: Benjamin WJ, editor. Borish's clinical refraction. Philadelphia: WS Saunders; 1998. p.179-241.

Bennett AG. The true founder of point-focal lens theory. Part I. Optician. 1965-a;150:395-8. 
Bennett AG. The true founder of point-focal lens theory. Part II. Optician, 1965-b;150:422-5.

Bennet AG, Rabbetts RB. Clinical visual optics. Boston: Butterwords; 1989. p.23-72.

Benjamin WJ. Visual optics of contact lens wear. In: Bennets ES, Eissman BA, editores. Clinical Contact Lens Practice. Philadelphia: JB Lippincott; 1991. p.1-42.

Benjamin WJ. Contact Lenses: clinical function and practical optics. In: Benjamin WJ, editor. Borish's clinical refraction. Philadelphia: WS Saunders; 1998. p.956-1021.

Castejón-Mochón JF, López-Gil N, Benito A, Artal P. Ocular wave-front aberrations statistics in a normal young population. Vis Res. 2002;42:1611-7.

Chalita MR, Chamon W, Schor P, Freda R, Krueger R. Resultados da literatura em tratamentos personalizados com Excimer Laser. In: Alves MR, Chamon W, Nose W, editores. Cirurgia refrativa. Rio de Janeiro: Cultura Médica; 2003. p.353-9.

Chamon W, Schor P, Carvalho LA, Castro JC. Aspectos básicos da Análise de Frente de Onda. In: Alves MR, Chamon W, Nose W, editores. Cirurgia refrativa. Rio de Janeiro: Cultura Médica; 2003. p.51-64.

Charman WN. Wavefront aberration of the eye: a review. Optom Vis Sci. $1991 ; 68,574-83$.

Collins MJ, Wildsoet CF, Atchison DA. Monochromatic aberrations and myopia. Vision Res. 1995;35(9):1157-63.

Cox I, Holden BA. Soft contact lenses-induced longitudinal spherical aberration and its effect on contrast sensitivity. Optom Vis Sci. 1990;67:67983. 
Davis JK. Geometric optics in ophthalmic lens designs. Proc Soc Photo-Opt Instrum Engl. 1973;39:65-100.

Doane JF, Scot M, Andrea B, Lon ES. Wavefront analysis. In: Boyd BF, Argarwal S, Argarwal A, Argarwal A, editores. LASIK and beyond LASIK. wavefront analysis customized ablation. Panama: Highlights of Ophtalmology; 2001. p.347-71.

Elliott DB. Contrast sensitivity and glare testing. In: Benjamin WJ. editor. Borish's clinical refraction. Philadelphia: WS Saunders: 1998. p.956-1021.

Grey CP. Changes in contrast sensitivity when wearing low, medium, and high water content soft lenses. J Br Contact Lens Assoc. 1986a;9:21-25.

Grey CP. Changes in contrast sensitivity during the first hour of soft contact lens wears. Am J Optom Physiol Opt. 1986b;63:702-7.

Guirao A, William DR, Cox IG. Effect of rotation and translation on the expected benefit of an ideal method to correct the eye's higher-order aberrations. J Opt Soc Am A Opt Image. Sci Vis. 2001;18:1003-15.

Guirao A, Porter J, William DR, Cox IG. Calculed impact of higher-order monochromatic aberrations on retinal image quality in a population of human eyes. J Opt Soc Am A Opt Image Sci Vis. $2002 ; 19$ :620-8.

Hament WJ, Nabar VA, Nuijts RMMA. Repeatability and validly of Zywave aberrometer measurements. J Cataract Refract Surg. 2002;28:2135-41.

He JC, Burns SdA, Marcos S. Monochromatic aberrations in the accommodated human eye. Vis Res. 2000;40:41-8.

Horner DG, Salmon TO, Soni OS. Corneal Topography. In: Benjamin WJ, editor. Borish's clinical refraction. Philadelphia: WS Saunders; 1998. p.52458. 
Howland HC, Howland B. A subjective method for the measurement of monochromatic aberrations of the human eye. J Opt Soc Am. 1977:67:150818.

Howland HC. The history and methods of ophthalmic wavefront sensing. $J$ Cataract Refract Surg. 2000;16:S552-S553.

Hulley SB, Newman TB, Cummings SR. Anatomia e fisiologia da pesquisa clínica. Porto Alegre: Artmed; 2003. p.22-34.

Jankov M, Rochen M, Schor P, Chamon W, Seiler T. Frentes de onda (Wavefronts) e limites da visão humana. Parte 1- Fundamentos. Arq Bras Oftalmol. 2002;65:679-84.

Jankov M, Schor P, Chamon W. Analisadores de frentes de onda. In: Alves MR, Chamon W, Nose W, editores. Cirurgia refrativa. Rio de Janeiro: Cultura Médica; 2003. p.183-91.

Katz M. The human eye as an optical system. In: Tasman W, Jaeger EE, editores. Duane's clinical ophthalmology. Philadelphia: Lippincott-Raven; 1981. v.1, cap. 33, p.1-52.

Kirkpatrick DL, Roggenkamp JR. Effects of soft contact lenses in contrast sensitivity. Am J Optom Phisiol, 1985;62:407-412.

Liang J, Grimm B, Goelz S, Bille JF. Objective measurement of wave aberrations of the human eye with the use of a Hartmann-Shack wave-front sensor. J Opt Soc Am A. 1994;11:1949-57.

Liang J, Williams DR. Aberrations and retinal image quality of the normal human eye. J Opt Soc Am A. 1997;14:2873-83.

Liang J, Williams DR, Miller D. Supernormal vision and high-resolution retinal imaging through adaptative optics. J Opt Soc Am A. 1997;14:2882-92. 
MacRae S, Fujieda M. Slit skiascopic-guided ablation using Nidek Laser. J Refract Surg. 2000;16:S576-S589.

Manns F, Ho A, Parel J, Culberstson W. Ablation profiles for wavefrontguided correction of primary spherical aberration. J Cataract Refract Surg. 2001;28:766-74.

Marcos S, Barbero S, Lhorente L, Merayo-Lhoves J. Optical response to LASIK surgery from total and corneal aberrations measurements. Invest Ophthalmol Vis Sci, 2001;42:3349-55.

Mirshahi A, Buren J, Gerhardt D, Kohnen T. In vivo and in vitro repeatability of Hartmann-Shack aberrometry. J Cataract Refract Surg. 2003;29:2295-302.

Mitra S, Lamberts DW. Contrast sensitivity in soft contact lens wearers. Contact Intraoocular Lens Med J. 1981;7:315-22.

Moreno-Bariuso E, Lhoves JM, Marcos S, Navarro R, Lhorente L, Barbero S. Ocular aberrations before and after myopic corneal refractive surgery: LASIK-induced changes measured with laser ray tracing. Invest Ophthalmol Vis Sci. 2001;42:1036-43.

Molebny VV, Panagopoulou SI, Molebny SV. Principles of Ray tracing aberrometry. J Refract Surg. 2000;16:S572-S575.

Morgan NW. Performance of ophthalmic lenses. J Am Optom Assoc. 1978;32:797-906.

Mrochen M, Kaemmerer M, Seiler T. Wavefront-guided Laser in situ Keratomileusis: early results in three eyes. J Cataract Refract Surg. 2000;16:116-21.

Oshika T, Klyce SD, Aplegate RA, Howland HC, Danasoury A. Comparison of corneal wavefront aberrations after Photorefractive and Laser in situ keratomileusis. Am J Ophthalmol. 1999;127:1-7. 
Porter J, Guirao A, Cox IG, Williams DR. Monochromatic aberrations of the human eye in a large population. J Opt Soc Am A Opt Image Sci Vis. 2001;18:1793-803.

Prado D. Noções de Óptica. Refração e adaptação de óculos. São Paulo: Revistas Técnicas; 1983. p.123-31.

Rudy MMA, Nuijts MD, Nabar VA, Hament WJ. Wavefront-guided versus standard laser in situ keratomileusis to correct low to moderate myopia. $J$ Cataract Refract Surg. 2002;28,1907-13.

Schimitzek T, Wasemann W. Clinical evaluation of refraction using a handheld wavefront autorefractor in young and adults patients. $J$ Cataract Refract Surg, 2002; 28:1655-66.

Sekuler R, Owsley C, Berenberger R. Contrast sensitivity during provoked visual impairment in multiple sclerosis. Opthal Phisiolol Opt. 1986;6:229-32.

Smirnov HS. Measurement of the wave aberration of the human eye. Biophysics. 1961;6:52-66.

Stein HA, Freeman JM, Stenson SM. Guia CLAO para refração e óculos. Um manual para oftalmologistas. New Orleans: CLAO; 1999. p.29-35.

Stephens GL. Correction with single vision spectacle lenses. In: Benjamin WJ, editor. Borish's clinical refraction. Philadelphia: WS Saunders; 1998. p.823-82.

Stephens GL, Davis JK. Spectacle lenses. In: Tasman WJ, Jaeger EA, editores. Duane's clinical ophthalmology. Philadelphia: Lippincott-Raven; 1993. v.1, cap.51, p.1-64.

Thibos LN. Principles of Hartmann-Shack Aberrometry. J Cataract Refract Surg. 2000;16:S563-565. 
Thibos LN, Hong X. Clinical applications of Hartmann-Shack Aberrometry. Optom Vis Sci. 1999;76:817-25.

Thibos LN, Hong X, Bradley A, Cheng X. Statistical variation of aberration structure and image quality in a normal population of healthy eyes. $J$ Opt Soc Am Opt Image Sci Vis. 2002 ;19:2329-49.

Tunnacliffe AH. Ocular aspects of vision. Introduction to visual optics. Londres: Association of British Dispensing Opticians, 1987. cap.7, p.402-12.

Tscherning M. Die monocrhomatischen aberrationene des menschlichen. Auges Z. Psychol Sinne. 1894;6:456-71. Apud Jankov M, Schor P, Chamon W. Analisadores de frentes de onda. In: Alves MR, Chamon W, Nose W, editores. Cirurgia refrativa. Rio de Janeiro: Cultura Médica; 2003. p.183-91.

Wang L, Misra M, Pallikaris IG, Koch DD. A comparison of a ray-tracing refractometer, autorefractor, and computerized videokeratography in measuring pseudophakik eyes. J Cataract Refract Surg. 2002;28:276-82.

Wang L, Koch DD. Ocular high-order aberrations in individuals screened for refractive surgery. J Cataract Refract Surg, 2003;29:1896-903.

Wachler BSB, Phillips CL, Schanzlin DJ, Krueger RR Comparison of Contrast Sensitivity in different soft contact lenses and spectacles. CLAO J. 1999;25:48-52.

Williams D, Yoon G, Porter J, Guirao A. Visual benefit of correcting high order aberrations of the eye. J Cataract Refract Surg. 2000;26:554-9.

Wo G, Hess R. Contrast sensitivity function and soft contact lenses. Int Contact Lens Clin. 1979;6:171-6.

Wolkstein M, Atkin A, Bodis-Wolmer A. Contrast sensitivity in retinal disease. Ophthalmology. 1980;87:1140-9. 
9 Fontes de consulta 
Bicas HE. Erros vernáculos mais freqüentes cometidos no meio médico acadêmico - parte 1. Arq Bras Oftalmol. 1999;62:229-33.

Dória Filho, U. Introdução à bioestatística para simples mortais. São Paulo: Negócios; 1999. 152p.

Hulley SB, Cummings SR, Browner WS, Grady D, Hearst N, Newman TB. Delineando a pesquisa clínica. Uma abordagem epidemiológica. Porto Alegre: Artmed; 2003. 374p.

Universidade de São Paulo. Faculdade de Medicina. Serviço de Biblioteca e Documentação. Guia de apresentação de dissertações, teses e monografias da FMUSP. Elaborado por Anneliese Carneiro da Cunha, Maria Julia A.L. Freddi, Maria F. Crestana, Marinalva de S. Aragão, Suely C. Cardoso, Valéria Vilhena. São Paulo: Serviço de Biblioteca e Documentação, 2004. 